\title{
Collective decision-making on networked systems in presence of antagonistic interactions
}

Angela Fontan

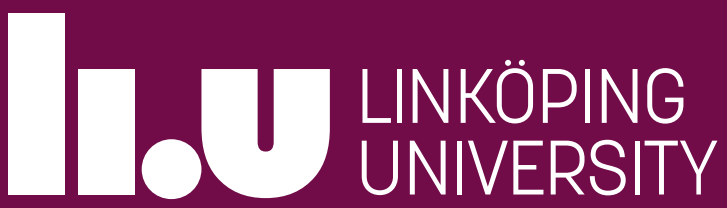



Linköping studies in science and technology. Dissertations.

No. 2166

\section{Collective decision-making on networked systems in presence of antagonistic interactions}

\section{Angela Fontan}


$((c)$ EY-NG This work is licensed under a Creative Commons AttributionNonCommercial 4.0 International License.

https://creativecommons.org/licenses/by-nc/4.0/

Linköping studies in science and technology. Dissertations.

No. 2166

Collective decision-making on networked systems in presence of antagonistic interactions

\author{
Angela Fontan \\ angela.fontan@liu.se \\ www. control.isy.liu.se \\ Division of Automatic Control \\ Department of Electrical Engineering \\ Linköping University \\ SE-581 83 Linköping \\ Sweden
}

ISBN 978-91-7929-017-7ＩSSN 0345-7524

Copyright @ 2021 Angela Fontan

Printed by LiU-Tryck, Linköping, Sweden 2021 
Ad Anna, Gianna e Luigi 



\section{Abstract}

Collective decision-making refers to a process in which the agents of a community exchange opinions with the objective of reaching a common decision. It is often assumed that a collective decision is reached through collaboration among the individuals. However in many contexts, concerning for instance collective human behavior, it is more realistic to assume that the agents can collaborate or compete with each other. In this case, different types of collective behavior can be observed. This thesis investigates collective decision-making problems in multiagent systems, both in the case of collaborative and of antagonistic interactions.

The first problem studied in the thesis is a special instance of the consensus problem, denoted "interval consensus" in this work. It consists in letting the agents impose constraints on the possible common consensus value. It is shown that introducing saturated nonlinearities in the decision-making dynamics to describe how the agents express their opinions effectively allows the agents to influence the achievable consensus value and steer it to the intersection of all the intervals imposed by the agents.

A second class of collective decision-making models discussed in the thesis is obtained by replacing the saturations with sigmoidal nonlinearities. This nonlinear interconnected model is first investigated in the collaborative case and then in the antagonistic case, represented as a signed graph of interactions. In both cases, it is shown that the behavior of the model can be described by means of bifurcation analysis, with the equilibria of the system encoding the possible decisions for the community. A scalar positive parameter, denoted "social effort", is added to the model to represent the strength of commitment between the agents, and plays the role of bifurcation parameter in the analysis. It is shown that if the social effort is small, then the community is in a deadlock situation (i.e., no decision is taken), while if the agents have the "right" amount of commitment two alternative consensus decision states for the community are achieved. However, by further increasing the social effort, the agents may fall in a situation of "overcommitment" where multiple (more than 2) decisions are possible. When antagonistic interactions between the agents are taken into account, they may lead to conflicts or social tensions during the decision-making process, which can be quantified by the notion of "frustration" of the signed network representing the community. The aim is to understand how the presence of antagonism (represented by the amount of frustration of the signed network) influences the collective decision-making process. It is shown that, while the qualitative behavior of the system does not change, the value of social effort required from the agents to break the deadlock (i.e., the value for which the bifurcation is crossed) increases with the frustration of the signed network: the higher the frustration, the higher the required social commitment.

A natural context to apply these results is that of political decision-making. In particular it is shown in the thesis how the government formation process in parliamentary democracies can be modeled as a collective decision-making system, where the agents are the parliamentary members, the decision is the vote of confidence they cast to a candidate cabinet coalition, and the social effort parameter is a proxy for the duration of the government negotiation talks. A signed network 
captures the alliances/rivalries between the political parties in the parliament. The idea is that the frustration of the parliamentary networks should correlate well with the duration of the government negotiation, and it is supported by the analysis of the legislative elections in 29 European countries in the last 40 years.

The final contribution of this thesis is an analysis of the structure of (signed) Laplacian matrices and of their pseudoinverses. It is shown that the pseudoinverse of a Laplacian is in general a signed Laplacian, and in particular that the set of eventually exponentially positive Laplacian matrices (i.e., matrices whose exponential is a matrix with negative entries which becomes and stays positive at a certain power) is closed under stability and matrix pseudoinversion. 


\section{Populärvetenskaplig sammanfattning}

Kollektivt beslutsfattande kan definieras som en process där agenter i en grupp utbyter åsikter med målet att fatta ett beslut. Det antas ofta att ett kollektivt beslut nås genom samarbete mellan individerna. I många fall, till exempel mänskligt kollektivt beteende, är det däremot mer realistiskt att anta att agenterna kan både samarbeta och motverka varandra. I dessa fall kan olika typer av kollektivt beteende observeras. Denna avhandling undersöker kollektivt beslutsfattande i fleragentssystem, både med kollaborativa och antagonistiska interaktioner.

Först studeras "intervallkonsensus", ett särskilt fall av konsensusproblemet där agenterna tillåts begränsa det möjliga konsensusvärdet. Det visas att genom att introducera begränsningar i hur agenterna uttrycker sina åsikter i beslutfattningsprocessen blir det möjligt för agenterna att påverka det möjliga konsensusvärdet och styra det till ett värde som accepteras av alla agenter.

En andra klass av kollektiva beslutfattningsmodeller som diskuteras i avhandlingen fås genom att ersätta de hårda begränsningarna med mjukare (S-formade olinjäriteter), och utöka modellen med en parameter kallad "social ansträngning" som representerar styrkan hos engagemanget mellan agenterna. Den sammankopplade modellen undersöks först i det kollaborativa fallet och sedan i det antagonistiska fallet. I grafen som beskriver gruppen representeras kollaborativa och antagonistiska interaktioner av bågar med positiva respektive negativa vikter. Målet är att förstå hur systemets beteende förändras när parametern för social ansträngning varieras. I båda fallen visas det att om den sociala ansträngningen är liten så hamnar gruppen i ett dödläge, men att om agenterna har lagom mängd engagemang så kan två alternativa konsensusbeslutstillstånd för gruppen uppnås. Genom att ytterligare öka den sociala ansträngningen kan agenterna däremot bli "överengagerade" vilket leder till fler än två möjliga beslut. När antagonistiska kopplingar mellan agenterna beaktas kan dessa leda till social spänning under beslutfattningsprocessen, vilket kan kvantifieras av "frustrationen" i det nätverk som representerar gruppen. Målet är att förstå hur närvaron av antagonism (representerad av mängden frustration) influerar den kollektiva beslutfattningsprocessen. Det visas att även om systemets kvalitativa beteende inte förändras, så ökar den mängd social ansträngning som krävs av agenterna för att bryta dödläget med frustrationen i nätverket: ju större frustration desto större socialt engagemang krävs.

Politiskt beslutsfattande är ett exempel där resultaten i avhandling kan tillämpas. Det visas hur regeringsbildningsprocessen i parlamentariska demokratier kan modelleras som ett kollektivt beslutfattningssystem där agenterna är parlamentarikerna, beslutet är förtroenerösten de ger till en kandiderande regeringskoalition, och den sociala ansträngningen representeras av regeringsförhandlingarnas längd. Parlamentet kan modelleras som en graf där noderna representerar parlamentarikerna och tecknet på bågarnas vikter representerar allianser och rivaliteter mellan deras respektive partier. Hypotesen är att frustrationen i det parlamentariska nätverket korrelerar väl med regeringsförhandlingarnas längd, vilket stöds av en analys av valen i 29 europeiska länder under de senaste 40 åren.

Det sista bidraget i denna avhandling är en analys av strukturen hos teckengrafer (d.v.s. grafer som kan ha både positiva och negativa bågvikter), och av de 
associerade laplacematriser och deras pseudoinverser. Pseudoinversen av en laplacematris har många tillämpningar vilket gör det viktigt att förstå dess egenskaper. Det visas att pseudomatrisen generellt är en laplacematris svarandes mot teckengraf, och villkor som garanterar stabilitet föreslås. 


\section{Acknowledgments}

The first person I need to thank is definitely my supervisor, Claudio Altafini. I am incredibly grateful for the opportunity of pursuing a $\mathrm{PhD}$ with you, your curiosity and passion for research are truly inspiring. Thank you for all your guidance and support, and for being available every time I needed help or got stuck. Grazie!

I would also like to thank my co-supervisor, Anders Hansson, for his support and for always having kind words towards me, and for the many discussions on our shared interests, such as music, opera, gardening, and much more.

I am grateful to Svante Gunnarsson, Martin Enqvist and Ninna Stensgård for providing a motivating, functioning and constructive work environment. To Alberto, Erik, Martin L., Gustaf, Jonatan and Anna, and all my colleagues at RT, thank you. Doing a PhD might feel lonely sometimes, and I am glad I have met so many nice, fun, and supportive colleagues and friends. Lunch walks, fika discussions, exchange of ideas at the reading group, workouts, dinners, BBQs, parties; in many occasions I have felt lucky to be part of the RT group. I treasure these memories. Kristoffer, Per, and Gustaf, I really appreciate all the help you have given me with thesis related issues in the last period.

I would also like to thank my friends outside RT. Elisabeth, I so appreciate our discussions and dinners (with cake and wine of course); Oskar, thank you for the lunches and for teaching me Swedish customs. Many thanks also to France (Alessandro) and Giulio, for all the great times we have shared together since university, and to Arianna, Mattia, and la compagnia di Breda, for welcoming me so warmly every time I return to Italy.

Finally, I want to thank my family. Thank you Olle, for being so supportive, caring and patient. I can't help but smile when I think about you. Un grazie speciale va ad Anna, Gianna e Luigi, per avermi sempre supportato (e sopportato direi) nei miei studi e sostenuto nelle mie scelte. Papà, per avermi insegnato a guardare il mondo con curiosità, ti penso spesso; mamma, per essere un ottimo esempio di donna forte e determinata; Anna, perchè so che posso sempre contare su di te (qui ci starebbe bene uno sticker).

Thank you all!

Linköping, August 2021 Angela Fontan 



\section{Contents}

\section{Background}

1 Introduction $\quad 3$

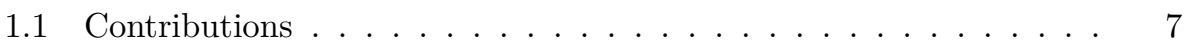

1.2 Thesis outline . . . . . . . . . . . . . . . . . . . 8

1.2.1 Part I: Background . . . . . . . . . . . . . . . 8

1.2.2 Part II: Publications . . . . . . . . . . . . . . 8

2 Concepts from Matrix Theory and Perron-Frobenius Theory 13

2.1 Elements of matrix theory . . . . . . . . . . . . . . . . . 13

2.2 Perron-Frobenius Theory ................. 15

3 Nonlinear systems $\quad 19$

3.1 Preliminary definitions . . . . . . . . . . . . . . . . 19

3.1.1 Cooperativity and Monotonicity .......... . . 23

3.2 Bifurcation analysis . . . . . . . . . . . . . . . . 24

3.2.1 Continuous-time systems: pitchfork bifurcation . . . . . . 25

3.2.2 Discrete-time systems: pitchfork and period-doubling bifurcations ..................... 27

4 Theory of graphs and social networks 31

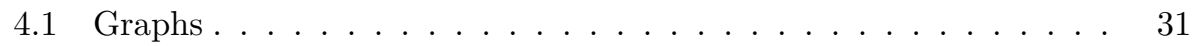

4.1.1 Laplacian of a graph and its properties . . . . . . . . . . 33

4.2 Signed graphs . . . . . . . . . . . . . . . . . . . . . . . 34

4.2.1 Signed Laplacian matrices . . . . . . . . . . . . . . . . . . . 34

4.3 Social networks as signed graphs . . . . . . . . . . . . . . 37

4.3.1 Structurally balanced graphs and frustration index . . . . . 37

5 Concluding remarks $\quad 41$

$\begin{array}{ll}\text { Bibliography } & 45\end{array}$ 


\section{Publications}

A Interval Consensus for Multiagent Networks $\quad \mathbf{5 3}$

1 Introduction . . . . . . . . . . . . . . . . . . 55

2 Problem Definition . . . . . . . . . . . . . . 58

2.1 The Model . . . . . . . . . . . . . . . . . . 58

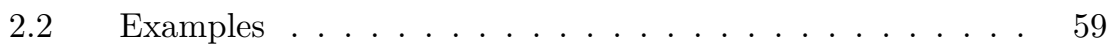

2.3 Paper Outline . . . . . . . . . . . . . . . . 59

3 Background Material . . . . . . . . . . . . . . . . 60

3.1 Cooperativity ..................... 60

3.2 Limit Set, Dini Derivatives, and Invariance Principle . . . . 61

3.3 Robust Consensus . . . . . . . . . . . . . . 62

4 Nonempty Interval Intersection: Interval Consensus . . . . . . . . . 62

4.1 Proof of Theorem $1 \ldots \ldots$. . . . . . . . . . 63

5 Empty Interval Intersection: Existence and Stability of Equilibria . 65

5.1 Proof of Theorem $2 \ldots \ldots 66$

5.2 Proof of Theorem $3 \ldots \ldots \ldots 67$

5.3 Proof of Theorem $4 \ldots \ldots \ldots$. . . . . . . . . . . . 69

5.4 Proof of Theorem $5 \ldots \ldots \ldots 72$

6 Nonempty Interval Intersection in Discrete-Time . . . . . . . . . . 73

7 Numerical Examples . . . . . . . . . . . . . . . . . . . . . 77

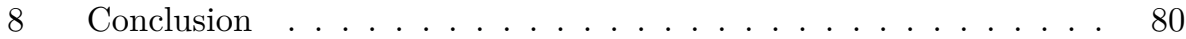

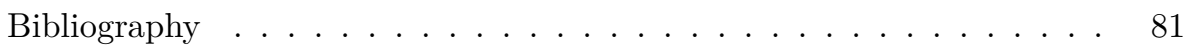

B Multiequilibria analysis for a class of collective decision-making $\begin{array}{ll}\text { networked systems } & \mathbf{8 5}\end{array}$

1 Introduction . . . . . . . . . . . . . . . . . 87

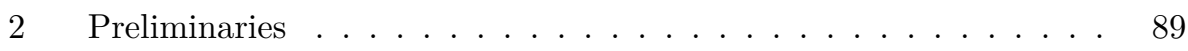

2.1 Concave and convex functions . . . . . . . . . . . 89

2.2 Nonnegative matrices and Perron-Frobenius . . . . . . . . . 90

2.3 Symmetric, symmetrizable and congruent matrices . . . . . 90

2.4 Cooperative systems . . . . . . . . . . . . . . 91

3 Multiple equilibria in collective decision-making systems . . . . . . 91

3.1 Existence of multiple equilibria: a necessary condition . . . 92

3.2 A geometric necessary and sufficient condition . . . . . . 96

3.3 Stability properties of multiple equilibria . . . . . . . . 99

4 Location of the mixed-sign equilibria . . . . . . . . . . . . . . . . 100

5 Numerical Analysis . . . . . . . . . . . . . . . . . . 101

6 Final considerations and conclusions . . . . . . . . . . . . . 104

Bibliography .................... 106

C The role of frustration in collective decision-making dynamical $\begin{array}{ll}\text { processes on multiagent signed networks } & 109\end{array}$

1 Introduction . . . . . . . . . . . . . . . . . 111

2 Preliminaries ........................... 114

$2.1 \quad$ Notation and linear algebra . . . . . . . . . . . 114 
$2.2 \quad$ Signed graphs . . . . . . . . . . . . . . . . . . 114

2.3 Monotone systems . . . . . . . . . . . . . . . 115

3 Decision-making in antagonistic multiagent systems in continuous-

time ...................... 116

3.1 Problem formulation . . . . . . . . . . . . . . 116

$3.2 \quad$ Structurally balanced case . . . . . . . . . . . . . . . . . 117

3.3 Structurally unbalanced case . . . . . . . . . . . . 117

4 Discrete-time . . . . . . . . . . . . . . . . . . 119

5 Discussion and interpretation of the results . . . . . . . . . . . . 122

6 Numerical Examples . . . . . . . . . . . . . . . . . . . . . . 124

7 Conclusions ........................... 130

A Technical preliminaries . . . . . . . . . . . . . . 130

B Proof of Theorem 2 . . . . . . . . . . . . . . 131

B.1 Proof of Theorem 2(i) . . . . . . . . . . . . 132

B.2 Proof of Theorem 2(ii.1): existence . . . . . . . . . 132

B.3 Proof of Theorem 2(ii.2): stability . . . . . . . . . . . 134

B.4 Proof of Theorem 2(ii.3): uniqueness . . . . . . . . . . 137

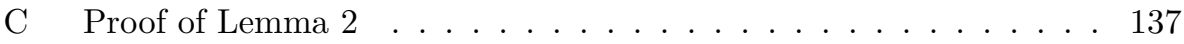

D Proof of Theorem 3.................... 138

D.1 Proof of Theorem 3(i) . . . . . . . . . . . . 139

D.2 Proof of Theorem 3(ii) . . . . . . . . . . . . . 141

E Proof of Proposition 1 . . . . . . . . . . . . . . . . . . . 142

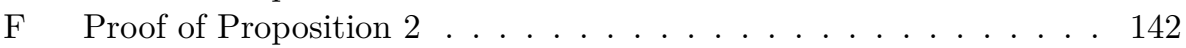

G Proof of Lemma 3 and Lemma 4 . . . . . . . . . . . . . 143

H Proof of Theorem 4................... 144

H.1 Proof of Theorem 4(i) . . . . . . . . . . . . . . 144

H.2 Proof of Theorem 4(ii) . . . . . . . . . . . . . 146

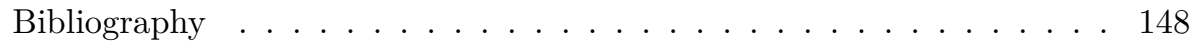

D A signed network perspective on the government formation pro$\begin{array}{lr}\text { cess in parliamentary democracies } & \mathbf{1 5 1}\end{array}$

1 Introduction . . . . . . . . . . . . . . . . . . . . 153

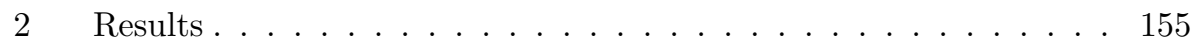

2.1 Correlation between frustration and duration of the government negotiation talks . . . . . . . . . . . . 155

2.2 Prediction of the successful cabinet coalition . . . . . . 158

2.3 Interpretation as collective decision dynamics . . . . . . . . 159

3 Discussion . . . . . . . . . . . . . . . . . . . . 163

4 Materials and Methods . . . . . . . . . . . . . . . . 170

4.1 Data description . . . . . . . . . . . . . . 170

4.2 Construction of parliamentary networks . . . . . . . . 171

4.3 Measuring the frustration of signed parliamentary networks 172

4.4 Frustration vs fractionalization index . . . . . . . . . . 172

4.5 Minimum energy government coalition . . . . . . . . . . . 173

4.6 Dynamical model of government formation . . . . . . . . . 174 
4.7 Rationale behind the correlation between frustration and duration of the government negotiation phase . . . . . . . 175

4.8 Leave-one-out analysis for scenario III . . . . . . . . . . . . 175

4.9 Yearly trends . . . . . . . . . . . . . . 176

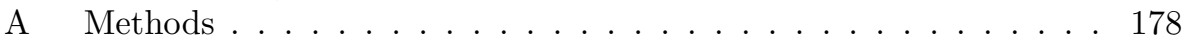

A.1 Parliamentary network construction . . . . . . . . . 178

A.2 Structural balance and frustration for signed networks . . . 181

A.3 Dynamical model of decision-making in presence of frustra-

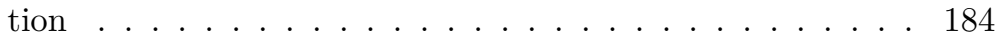

B Application: from parliamentary networks to government formation 187

B.1 Frustration vs government negotiation days . . . . . . . . 187

B.2 Minimum energy government coalition . . . . . . . . . . 187

B.3 Frustration vs smallest eigenvalue of the normalized signed Laplacian . . . . . . . . . . . . . . . . . . . . . . 189

B.4 Frustration vs fractionalization index . . . . . . . . . 190

B.5 Description of the results . . . . . . . . . . . 192

B.6 Influential points of the regression are important . . . . . . 192

B.7 A brief discussion on national rules and traditions influencing the duration of the government negotiation talks . . . . 193

B.8 A brief discussion on elections resulting in a hung parliament 194

B.9 A brief discussion on elections where the government negotiations failed . . . . . . . . . . . . . . 195

B.10 Analysis of the type of governments formed after the elections 196

B.11 Analysis of the Italian bicameral parliamentary system . . . 197

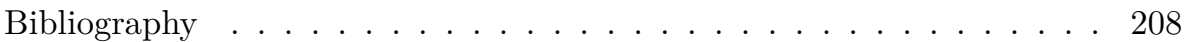

E On the properties of Laplacian pseudoinverses $\quad \mathbf{2 1 5}$

1 Introduction . . . . . . . . . . . . . . . . . 217

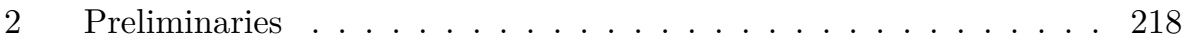

$2.1 \quad$ Linear algebraic preliminaries . . . . . . . . . . . . . . 218

2.2 Signed graphs . . . . . . . . . . . . . . . . . . 219

2.3 Eventual exponential positivity . . . . . . . . . . . 220

2.4 Kron reduction for undirected networks . . . . . . . . . 220

$3 \quad$ Pseudoinverse of eventually exponentially positive Laplacians . . . 221

3.1 Directed signed network case . . . . . . . . . . . . 221

3.2 Undirected signed network case . . . . . . . . . . . . . 227

4 Electrical networks and effective resistance . . . . . . . . . . . . . 228

5 Conclusions and future work . . . . . . . . . . . . . . . 231

Bibliography ..................... . . 232 
Part I

\section{Background}





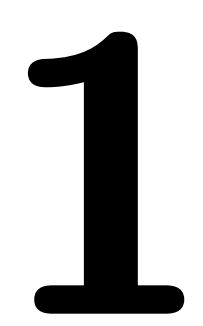

\section{Introduction}

The idea of networks of interacting agents exchanging opinions or preferences in order to achieve a common decision as a community is not specific of a particular field or discipline, and examples of collective behaviors can be observed in everyday life. Typical examples range from collective behaviors in animal groups, such as selection of nest location, food source or migration mechanisms [1-4], to coordination and formation control or task-allocation problems in robotics [5-7], to opinion formation $[8,9]$. In many of these cases, an agreement is reached through collaboration between the agents. However, there are other scenarios, such as e.g. social networks, trade markets, sport games, or parliamentary systems, where competition among the agents is intrinsic and unavoidable [10], hence the outcome of the interaction is less clear and the fact of achieving some form of agreement is not guaranteed.

Focusing on opinion dynamics models, i.e., on exchange of opinions among agents on a "social network", in order to describe a collective decision-making process two elements are required: a framework able to capture how the individuals represent their opinions and communicate with each other, and a dynamical model describing how the opinions of the agents evolve in time. The natural choice for the framework is a state space model with state variables representing the opinions and a graph representing the interactions among the agents. As for the dynamics, many choices have been proposed in the literature in the last fifty years, as reviewed for instance in $[11,12]$, ranging from linear to nonlinear, from continuous- to discretetime, from deterministic to probabilistic, etc.

Given a community of $n$ agents, represented as a network $\mathcal{G}$, a state variable $x_{i}(t) \in \mathbb{R}$ (to ease the notation, the time-dependence is omitted hereafter) is assigned to each node $i$ of the network to represent the opinion of agent $i$ (at time $t$ ). In continuous-time, the evolution of the opinions of the agents in the network 
$\mathcal{G}$ can be described by a dynamical model in the generic form

$$
\dot{x}=f(x)=\left[f_{1}(x) \cdots f_{n}(x)\right]^{T}
$$

where $x=\left[x_{1} \cdots x_{n}\right]^{T} \in \mathbb{R}^{n}$ is the vector of opinions, and $f: \mathbb{R}^{n} \rightarrow \mathbb{R}^{n}$ is a vector field which depends on the structure of the network $\mathcal{G}$. The possible decisions for the community can be encoded in the equilibria of the system. When $f(x)=-L x$, where $L$ is the Laplacian matrix of the network $\mathcal{G}$, then the equilibria of the system (1.1) have all equal components. These types of equilibria are called consensus, and have been extensively studied in the literature, see e.g. [6, 11, 13].

Linear models like the one leading to consensus may fail to capture more complex behaviors, and nonlinearities are sometimes included in an attempt to make the models more realistic. However, the adoption of nonlinear models means that the dynamics may exhibit new phenomena, such as multiple equilibria, periodic orbits, and bifurcations. For instance, a class of nonlinear interconnected models that combines consensus with saturated and sigmoidal nonlinearities is proposed in [4]. It has the following structure:

$$
\dot{x}=-\Delta x+\pi A \psi(x)
$$

where $A=\left[a_{i j}\right] \in \mathbb{R}^{n \times n}$ is the adjacency matrix of the graph $\mathcal{G}, \Delta=\operatorname{diag}\left\{\delta_{1}, \ldots, \delta_{n}\right\}$, with $\delta_{i}=\sum_{j=1}^{n} a_{i j}$ for all $i$, is the diagonal term representing the weighted indegree, $\pi>0$ is a positive scalar parameter, and $\psi(x)=\left[\psi_{1}\left(x_{1}\right) \cdots \psi_{n}\left(x_{n}\right)\right]^{T}$ is a vector of sigmoidal and saturated nonlinearities, see Fig. 1.1. By construction, in this model the linearization at the origin (for $\pi=1$ ) corresponds to the graph Laplacian $L=\Delta-A$. The model (1.2) is considered in paper A of part II of this thesis for the case of saturation nonlinearities, and in paper B for the case of sigmoidal nonlinearities.

The interaction graph $\mathcal{G}$ considered in the model (1.1) (and (1.2)) typically has edges with nonnegative weights, which capture the fact that the agents are collaborating. A natural way to extend this model to the case of agents that compete is to consider signed graphs, i.e., graphs whose edges have positive or negative weight [14]: a positive edge indicates cooperation (or friendship, trust) while a negative edge competition (or antagonism, distrust). A notion that is often used when dealing with signed graphs is that of structural balance, which captures the idea that no social tension is present at a network level even if antagonism is present (at a local level) between the agents. In particular, a signed graph is structurally balanced if its agents can be divided into two subgroups such that the agents in each subgroup are mutual friends (meaning that they are linked by edges with positive weight), and the agents belonging to different subgroups are rivals (linked by edges with negative weight) $[15,16]$. A closely related notion is that of frustration of a signed graph, a concept introduced to measure its distance from a structurally balanced state $[17,18]$ (see also [19] for an overview of the different measures proposed in the literature to estimate the level of imbalance a of signed graph): a signed graph is structurally balanced if and only if it has zero frustration.

In the context of multiagent dynamics, the notion of structural balance is strictly related to that of monotone systems (or order preserving systems) [20], and 


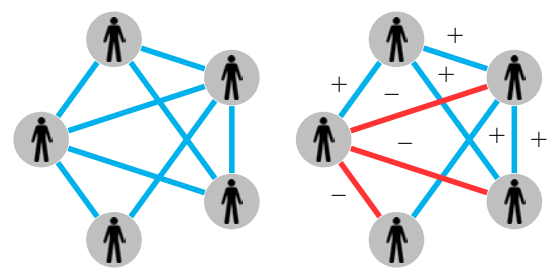

(a)
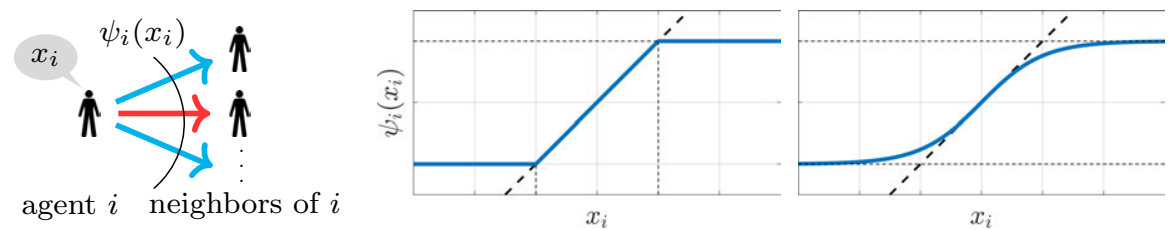

(b)

Figure 1.1: Representation of a collective decision-making process, described as a nonlinear system over (signed) networks, see (1.2). (a): A community of agents is modeled as a graph of interactions, which may be unsigned/cooperative (left) or signed (right). (b): Nonlinear functions $\psi_{i}(\cdot), i=1, \ldots, n$, represent how each agent $i$ transmits its opinion $x_{i}$ to its neighbors in the network. The nonlinearities considered in this work are saturated and monotonically increasing.

the notion of frustration to that of distance to monotonicity [21]. In particular, for nonnegative graphs $\mathcal{G}$ with saturated/sigmoidal nonlinearities, the model (1.2) is a so-called cooperative system, while when $\mathcal{G}$ is signed and structurally balanced then (1.2) is a monotone system.

Real-world signed networks, from e.g., biological networks [18, 21, 22] to social networks [23, 24] and (multi-party) parliamentary networks [25], are in general not structurally balanced. For this reason, it is relevant to understand how the antagonism present in the signed networks affects the collective decision-making process. In paper $\mathrm{C}$ of part II of this thesis, the model (1.2) is extended to include the case in which the graph $\mathcal{G}$ is signed. The difference is that the adjacency matrix $A$ of $\mathcal{G}$ is now a signed matrix, with the sign of each element $(+1$ or -1$)$ representing the type of interaction (friendly/trust/alliance or unfriendly/distrust/rivalry), and the absolute value the amount of trust/distrust between the corresponding agents. Similarly, $\Delta=\operatorname{diag}\left\{\delta_{1}, \ldots, \delta_{n}\right\}$ is still a weighted in-degree matrix, but now with $\delta_{i}=\sum_{j=1}^{n}\left|a_{i j}\right|$ for all $i$. Under these assumptions, the linearization of the model (1.2) at the origin corresponds to a signed version of the Laplacian [16]. In particular, the system (1.2) is monotone if and only if the graph $\mathcal{G}$ is structurally balanced. Consequently, when $\mathcal{G}$ is not structurally balanced, its dynamics is not monotone and hence in general more complicated to understand. In papers $\mathrm{C}$ and $\mathrm{D}$ it is shown that the notion of frustration can be used to get some insight into the behavior of the system (1.2). 


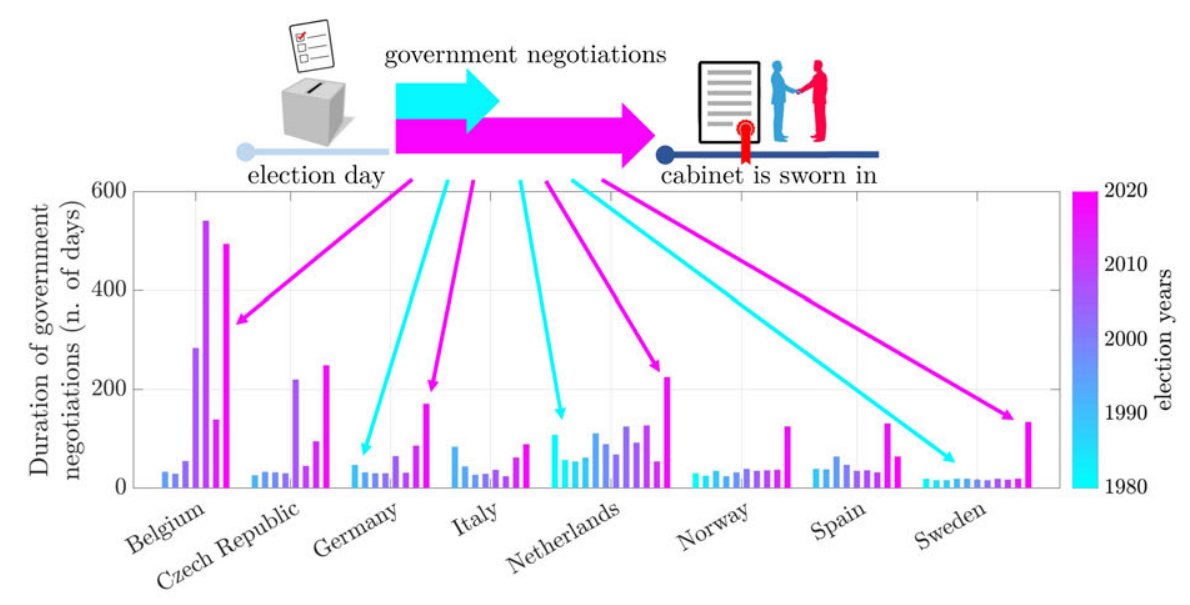

Figure 1.2: Duration of government negotiations (calculated as number of days between the election date and the date the government is sworn in) in selected European countries in the last 40 years (see paper D).

\section{Example: Political Decision-Making}

The government formation process in countries with a parliamentary system is a good example of collective decision-making applied to the field of Political Sciences. Briefly, the process starts with a legislative election after which each political party receives a certain number of seats in the parliament, depending on the number of votes gained at the election. In case of no clear winner, i.e., when no party or coalition has managed to secure a majority in the parliament, a negotiation starts between the political parties. The process concludes with the swearing-in of a new government, typically after winning a confidence vote in the parliament. The government negotiation phase might be complex and long. For instance, the past ten years have seen an increase in the duration of government negotiations, defined as the number of days between the election date and the date the government is sworn in. This has happened not only in countries famous for long periods of cabinet negotiations, such as Belgium, but also, e.g., in Germany, UK, and Sweden, see Fig. 1.2. The research question considered in paper D of part II of this thesis work is whether it is possible to use models for collective decision-making to explain this behavior. The idea is to describe the government formation process as a collective decision-making system on a signed graph having the parliamentary members as agents and their alliances/rivalries as edges. The resulting graphs are not structurally balanced, and the hypothesis is that their frustration correlates with the duration of the government negotiation phase. 


\subsection{Contributions}

The contributions of this thesis work are within the area of complex (in general, signed) networks and nonlinear dynamics over networks, with applications to collective decision-making processes over social networks.

Starting from a problem of consensus proposed in Paper A, Papers B and C formulate a collective decision-making problem over cooperative and antagonistic networks, respectively, as a bifurcation problem, where the crossing of a pitchfork bifurcation corresponds to the achievement of a common decision. The results of Paper $\mathrm{C}$ admit a natural interpretation in the context of social networks, and in Paper $\mathrm{D}$ a concrete example of political decision-making is proposed. In paper $\mathrm{E}$, departing from the study of nonlinear dynamics over networks, the focus is only on signed graphs and in particular on the structure of the signed Laplacian matrix and its pseudoinverse.

More specifically, the main contributions of this thesis work are:

- Formulation of a nonlinear interconnected cooperative model for "interval consensus", where nonlinearities are introduced to allow the agents to impose constraints on the achievable consensus value. If the intersection of the constraints is nonempty, convergence to interval consensus is proven. (Paper A)

- Analysis of a nonlinear interconnected cooperative model for collective decisionmaking with saturating and sigmoidal nonlinearities, and scalar bifurcation parameter. Necessary and sufficient conditions for existence and stability of equilibria are proposed, and an exact upper bound for the norm of equilibria is derived. (Paper B)

- Extension of the nonlinear model of Paper B to antagonistic networks. Analysis of the qualitative behavior of the system, both in continuous- and discrete-time. (Paper C)

- Interpretation of the results of Paper $\mathrm{C}$ in terms of frustration of the signed network representing the community: the bifurcation value at which the first bifurcation is crossed is shown to depend on the frustration, and an upper bound for the 1-norm of the equilibria depending on the frustration is derived. (Paper C)

- Introduction of a concrete example of the theoretical work of paper C. The process of government formation in parliamentary democracies is explained as a collective decision-making process over signed "parliamentary" networks, where the crossing of a bifurcation corresponds to success/failure of a confidence vote. Through the collection and analysis of data of legislative elections in the last 40 years for 29 European countries, the frustration of the parliamentary networks is shown to be a good proxy for the complexity of the government negotiation process. (Paper D)

- Study of the properties of "repelling" signed Laplacian matrices and their pseudoinverses. The set of repelling signed Laplacian matrices which are eventually exponentially positive is shown to be closed under pseudoinversion and stability. (Paper E) 


\title{
1.2 Thesis outline
}

The thesis is divided into two parts, with background material in the first part, and edited versions of published papers in the second part.

\subsubsection{Part I: Background}

The first part introduces theoretical background for the publications in part II. The preliminary material includes concepts and notions from matrix and graph theory, and nonlinear systems.

\subsubsection{Part II: Publications}

The second part of this thesis contains edited versions of the five papers listed below.

\section{Paper A: Interval Consensus for Multiagent Networks}

Paper $\mathrm{A}$ is an edited version of

\begin{abstract}
A. Fontan, G. Shi, X. Hu, and C. Altafini, "Interval Consensus for Multiagent Networks," IEEE Transactions on Automatic Control, vol. 65, no. 5, pp. 1855-1869, may 2020.
\end{abstract}

Summary The constrained consensus problem considered in paper A, denoted interval consensus, is characterized by the fact that each agent can impose a lower and upper bound on the achievable consensus value. Such constraints can be encoded in the consensus dynamics by saturating the values that an agent transmits to its neighboring nodes. We show in the paper that when the intersection of the intervals imposed by the agents is nonempty, the resulting constrained consensus problem must converge to a common value inside that intersection. In our algorithm, convergence happens in a fully distributed manner, and without need of sharing any information on the individual constraining intervals. When the intersection of the intervals is an empty set, the intrinsic nonlinearity of the network dynamics raises new challenges in understanding the node state evolution. Using Brouwer fixed-point theorem we prove that in that case there exists at least one equilibrium, and in fact the possible equilibria are locally stable if the constraints are satisfied or dissatisfied at the same time among all nodes. For graphs with sufficient sparsity it is further proven that there is a unique equilibrium that is globally attractive if the constraint intervals are pairwise disjoint.

Contribution and background The author of this thesis contributed with implementations, analysis and reviewing the manuscript. 


\section{Paper B: Multiequilibria Analysis for a Class of Collective Decision-Making Networked Systems}

Paper B is an edited version of

A. Fontan and C. Altafini, "Multiequilibria Analysis for a Class of Collective Decision-Making Networked Systems," IEEE Transactions on Control of Network Systems, vol. 5, no. 4, pp. 1931-1940, dec 2018.

Summary The models of collective decision-making considered in paper B are nonlinear interconnected cooperative systems with saturating interactions. These systems encode the possible outcomes of a decision process into different steady states of the dynamics. In particular, they are characterized by two main attractors in the positive and negative orthant, representing two choices of agreement among the agents, associated to the Perron-Frobenius eigenvector of the system. In this paper we give conditions for the appearance of other equilibria of mixed sign. The conditions are inspired by Perron-Frobenius theory and are related to the algebraic connectivity of the network. We also show how all these equilibria must be contained in a solid disk of radius given by the norm of the equilibrium point which is located in the positive orthant.

Contribution and background The author of this thesis contributed with the majority of the work including theoretical derivations, implementations, numerical calculations and the written manuscript.

\section{Paper C: The role of frustration in collective decision-making dynamical processes on multiagent signed networks}

Paper $\mathrm{C}$ is an edited version of

A. Fontan and C. Altafini, "The role of frustration in collective decisionmaking dynamical processes on multiagent signed networks," arXiv:2105.11396, pp. 1-18, may 2021.

Summary In paper $\mathrm{C}$ we consider a collective decision-making process in a network of agents described by a nonlinear interconnected dynamical model with sigmoidal nonlinearities and signed interaction graph. The decisions are encoded in the equilibria of the system. The aim is to investigate this multiagent system when the signed graph representing the community is not structurally balanced and in particular as we vary its frustration, i.e., its distance to structural balance. The model exhibits bifurcations, and a "social effort" parameter, added to the model to represent the strength of the interactions between the agents, plays the role of bifurcation parameter in our analysis. We show that, as the social effort increases, the decision-making dynamics exhibits a pitchfork bifurcation behavior where, from a deadlock situation of "no decision" (i.e., the origin is the only globally stable equilibrium point), two possible (alternative) decision states for the community are achieved (corresponding to two nonzero locally stable equilibria). 
The value of social effort for which the bifurcation is crossed (and a decision is reached) increases with the frustration of the signed network.

Contribution and background The author of this thesis contributed with the majority of the work including theoretical derivations, implementations, numerical calculations and the written manuscript.

\section{Paper D: A signed network perspective on the government formation process in parliamentary democracies}

Paper D is an edited version of

A. Fontan and C. Altafini, "A signed network perspective on the government formation process in parliamentary democracies," Scientific Reports, vol. 11, no. 5134, dec 2021.

Summary In parliamentary democracies, government negotiations talks following a general election can sometimes be a long and laborious process. In order to explain this phenomenon, in this paper we use structural balance theory to represent a multiparty parliament as a signed network, with edge signs representing alliances and rivalries among parties. We show that the notion of frustration, which quantifies the amount of "disorder" encoded in the signed graph, correlates very well with the duration of the government negotiation talks. For the 29 European countries considered in this study, the average correlation between frustration and government negotiation talks ranges between 0.42 to 0.69 , depending on what information is included in the edges of the signed network. Dynamical models of collective decision-making over signed networks with varying frustration are proposed to explain this correlation.

Contribution and background The author of this thesis contributed with the majority of the work including theoretical derivations, implementations, numerical calculations and the written manuscript.

\section{Paper E: On the properties of Laplacian pseudoinverses}

Paper E is an edited version of
A. Fontan and C. Altafini, "On the properties of Laplacian pseudoinverses," in 60th IEEE Conference on Decision and Control. Austin, TX, USA: IEEE, 2021.

Summary The pseudoinverse of a graph Laplacian is used in many applications and fields, such as for instance in the computation of the effective resistance in electrical networks, in the calculation of the hitting/commuting times for a Markov chain and in continuous-time distributed averaging problems. In this paper we show that the Laplacian pseudoinverse is in general not a Laplacian matrix but rather a signed Laplacian with the property of being an eventually exponentially 
positive matrix, i.e., of obeying a strong Perron-Frobenius property. We show further that the set of signed Laplacians with this structure (i.e., eventual exponential positivity) is closed with respect to matrix pseudoinversion. This is true even for signed digraphs, and provided that we restrict to Laplacians that are weight balanced also stability is guaranteed.

Contribution and background The author of this thesis contributed with the majority of the work including theoretical derivations, implementations, numerical calculations and the written manuscript.

\section{Publications}

Works published by the author of this thesis are listed below in chronological order $^{1}$. Publications indicated by a $\star$ are included in the thesis.

A. Fontan, G. Shi, X. Hu, and C. Altafini, "Interval consensus: a novel class of constrained consensus problems for multiagent networks," in 56th IEEE Conference on Decision and Control. Melbourne, Australia: IEEE, dec 2017, pp. 4155-4160.

A. Fontan and C. Altafini, "Investigating mixed-sign equilibria for nonlinear collective decision-making systems," in 56th IEEE Conference on Decision and Control. Melbourne, Australia: IEEE, dec 2017, pp. 781-786.

$\star$ A. Fontan and C. Altafini, "Multiequilibria Analysis for a Class of Collective Decision-Making Networked Systems," IEEE Transactions on Control of Network Systems, vol. 5, no. 4, pp. 1931-1940, dec 2018.

A. Fontan and C. Altafini, "Modeling wireless power transfer in a network of smart devices: A compartmental system approach," in 2018 17th European Control Conference (ECC). Limassol, Cyprus: European Control Association (EUCA), 2018, pp. 1468-1473.

A. Fontan and C. Altafini, "Achieving a decision in antagonistic multi agent networks: frustration determines commitment strength," in 57th IEEE Conference on Decision and Control. Miami Beach, FL, USA: IEEE, dec 2018, pp. 109-114.

$\star$ A. Fontan, G. Shi, X. Hu, and C. Altafini, "Interval Consensus for Multiagent Networks," IEEE Transactions on Automatic Control, vol. 65, no. 5, pp. 1855-1869, may 2020.

$\star$ A. Fontan and C. Altafini, "A signed network perspective on the government formation process in parliamentary democracies," Scientific Reports, vol. 11, no. 5134, dec 2021.

\footnotetext{
${ }^{1}$ Extended abstracts are not included in the list.
} 
$\star$ A. Fontan and C. Altafini, "The role of frustration in collective decisionmaking dynamical processes on multiagent signed networks," arXiv:2105.11396, pp. 1-18, may 2021.

$\star$ A. Fontan and C. Altafini, "On the properties of Laplacian pseudoinverses," in 60th IEEE Conference on Decision and Control. Austin, TX, USA: IEEE, 2021. 


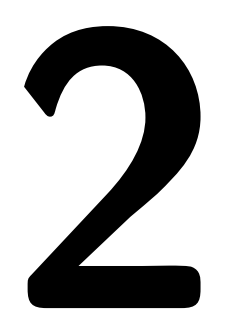

\section{Concepts from Matrix Theory and Perron-Frobenius Theory}

The aim of this chapter is to introduce notation and preliminary material from matrix theory, and it is mostly based on [34]. The second part of this chapter is dedicated to Perron-Frobenius theory. This topic is relevant for this thesis work due to its applications in dynamical systems (e.g., the study of cooperative/monotone systems) and graph theory (e.g., the study of the properties of Laplacian matrices).

\subsection{Elements of matrix theory}

This section presents a collection of definitions and results from matrix theory, with particular focus on the characterization of eigenvalues ${ }^{1}$.

Let $\mathbb{N}, \mathbb{R}, \mathbb{C}$ indicate the set of natural, real, and complex numbers, respectively. The spectrum of a matrix $A \in \mathbb{R}^{n \times n}$ is denoted $\Lambda(A)=\left\{\lambda_{1}(A), \ldots, \lambda_{n}(A)\right\}$, where $\lambda_{i}(A), i=1, \ldots, n$, are the eigenvalues of $A$. The eigenvalues are in general assumed to be arranged in the following (nondecreasing) order: $\operatorname{Re}\left[\lambda_{1}(A)\right] \leq \operatorname{Re}\left[\lambda_{2}(A)\right] \leq$ $\cdots \leq \operatorname{Re}\left[\lambda_{n}(A)\right]$, where $\operatorname{Re}[\cdot]$ denotes the real part (similarly, $\operatorname{Im}[\cdot]$ is used to denote the imaginary part). The spectral radius of $A$ is $\rho(A)=\max \{|\lambda|: \lambda \in \Lambda(A)\}$, and its spectral abscissa is $\mu(A)=\max \{\operatorname{Re}[\lambda]: \lambda \in \Lambda(A)\}$.

The kernel (or, null space) of $A$ is $\operatorname{ker}(A)=\left\{x \in \mathbb{R}^{n}: A x=0\right\}$, and its range (or, column space) is $\operatorname{range}(A)=\left\{y \in \mathbb{R}^{n}: y=A x\right\}$. The $\operatorname{rank}$ of $A$ is $\operatorname{rank}(A)=$ $\operatorname{rank}\left(A^{T}\right)=n-\operatorname{dim}(\operatorname{ker}(A))$, and the corank of $A$ is corank $(A)=\operatorname{dim}(\operatorname{ker}(A))$.

Characterization of symmetric and symmetrizable matrices $\mathrm{A}$ matrix $A \in$ $\mathbb{R}^{n \times n}$ is called symmetric if $A=A^{T}$, and it is called (diagonally, omitted hereafter) symmetrizable if $D A$ is symmetric for some diagonal matrix $D$ with positive

\footnotetext{
${ }^{1}$ Basically, the aim is to answer the questions: "if a matrix satisfies a certain property, what conclusions can be drawn on its eigenvalues?", or, similarly: "if two matrices satisfy a certain relationship, what conclusions can be drawn on their eigenvalues?"
} 
diagonal entries. Symmetric or symmetrizable matrices have real eigenvalues (see $[35,36]$ for a more detailed characterization of symmetrizable matrices).

Characterization of similar and congruent matrices Given two matrices $A, B \in$ $\mathbb{R}^{n \times n}, B$ is similar to $A$ (denoted $A \sim B$ ) if there exists a nonsingular matrix $S \in \mathbb{R}^{n \times n}$ such that $B=S^{-1} A S$, while it is congruent to $A$ if there exists a nonsingular matrix $S \in \mathbb{R}^{n \times n}$ such that $B=S A S^{T}$. Similar matrices have the same eigenvalues. Congruent matrices that are also symmetric have the same inertia, i.e., the same number (counting multiplicity) of positive, negative and zero eigenvalues, also known as Sylvester's law of inertia [34, Thm 4.5.8]. Moreover, a bound between the eigenvalues of congruent (and symmetric) matrices is provided by the Ostrowski's Theorem.

Theorem 2.1 (Ostrowski, 4.5.9 in [34]). Let $A, S \in \mathbb{R}^{n \times n}$ with A symmetric and $S$ nonsingular. Let the eigenvalues of $A, S A S^{T}$ and $S S^{T}$ be arranged in nondecreasing order. For each $k=1, \ldots, n$, there exists a positive real number $\theta_{k}$ such that $\lambda_{1}\left(S S^{T}\right) \leq \theta_{k} \leq \lambda_{n}\left(S S^{T}\right)$ and $\lambda_{k}\left(S A S^{T}\right)=\theta_{k} \lambda_{k}(A)$.

Characterization of diagonally dominant matrices A matrix $A=\left[a_{i j}\right] \in \mathbb{R}^{n \times n}$ is called diagonally dominant (by rows, omitted hereafter) if

$$
\left|a_{i i}\right| \geq \sum_{j=1}^{n}\left|a_{i j}\right|, \quad i=1, \ldots, n
$$

diagonally equipotent (nomenclature from [37]) if (2.1) holds with equality for all $i$, weakly diagonally dominant if (2.1) holds and at least one (but not all) of the inequalities is strict, and strictly diagonally dominant if (2.1) holds with strict inequality for all $i$. A matrix $A \in \mathbb{R}^{n \times n}$ is called irreducible if there does not exist a permutation matrix $P$ s.t. $P^{T} A P$ is block triangular.

Strictly diagonally dominant matrices are nonsingular, and, if all their diagonal elements are strictly positive, then their eigenvalues have positive real part, see [34, Thm 6.1.10 (Levy-Desplanques)]. The same characterization holds for matrices that are irreducibly diagonally dominant, i.e., matrices that are irreducible and weakly diagonally dominant, see [34, Corollary 6.2.27 (Taussky)]).

Diagonally equipotent matrices that are irreducible and have nonnegative diagonal elements, have eigenvalues with nonnegative real part, and their corank is at most 1 [37, Proposition 1].

The Geršgorin's Theorem is an useful tool to prove (or give the intuition behind) the aforementioned results.

Theorem 2.2 (Geršgorin, 6.1.1 in [34]). Let $A=\left[a_{i j}\right] \in \mathbb{R}^{n \times n}$. The eigenvalues of $A$ are in the union of the $n$ Geršgorin's disks, defined as

$$
\left\{\lambda \in \mathbb{C}:\left|\lambda-a_{i i}\right| \leq \sum_{j=1}^{n}\left|a_{i j}\right|\right\}, \quad i=1, \ldots, n
$$




\subsection{Perron-Frobenius Theory}

In 1907 Perron showed that the class of positive matrices (i.e., matrices whose elements are strictly positive) is characterized by an important property, namely, that the spectral radius of a positive matrix is an eigenvalue and that the corresponding (left and right) eigenvectors are strictly positive. Later, thanks to Frobenius, this result was extended to the class of irreducible and nonnegative matrices (i.e., matrices with nonnegative elements), and recently to the class of eventually positive matrices (i.e., matrices which become and stay positive after a certain power). These findings, as well as related results, are reported in this section.

Characterization of positive and nonnegative matrices $\mathrm{A}$ matrix $A=\left[a_{i j}\right] \in$ $\mathbb{R}^{n \times n}$ is positive (denoted $A>0$ ) if $a_{i j}>0$ for all $i, j=1, \ldots, n$, and is nonnegative (denoted $A \geq 0$ ) if $a_{i j} \geq 0$ for all $i, j=1, \ldots, n$. Similarly, a vector $x \in \mathbb{R}^{n}$ is positive (denoted $x>0$ ) if $x_{i}>0$ for all $i=1, \ldots, n$, and is nonnegative (denoted $x \geq 0$ ) if $x_{i} \geq 0$ for all $i=1, \ldots, n$.

A useful property is that the largest and smallest row sum of a nonnegative matrix provide an upper and lower bound, respectively, for its spectral radius.

Lemma 2.1 (Thm 8.1.22 in [34]). Let $A \in \mathbb{R}^{n \times n}$ be nonnegative. Then

$$
0 \leq \min _{i=1, \ldots, n}\left\{\sum_{j=1}^{n} a_{i j}\right\} \leq \rho(A) \leq \max _{i=1, \ldots, n}\left\{\sum_{j=1}^{n} a_{i j}\right\}
$$

Perron-Frobenius theory can be used to understand: (i) when the spectral radius is strictly positive, and (ii) when it is an eigenvalue.

The following theorem, called Perron's Theorem, characterizes the spectral properties of positive matrices.

Theorem 2.3 (Perron, 8.2.8 in [34]). Let $A \in \mathbb{R}^{n \times n}$ be positive. Then

(i) $\rho(A)>0$ is an algebraically simple eigenvalue of $A$;

(ii) there is a unique real vector $\chi \in \mathbb{R}^{n}$ such that $A \chi=\rho(A) \chi$, and $\chi>0$;

(iii) there is a unique real vector $\xi \in \mathbb{R}^{n}$ such that $\xi^{T} A=\rho(A) \xi^{T}$, and $\xi>0$;

(iv) $\rho(A)>|\lambda|$ for all $\lambda \in \Lambda(A)$;

(v) $\lim _{k \rightarrow \infty}\left(\rho(A)^{-1} A\right)^{k}=\chi \xi^{T}$.

In general, these properties do not hold for the class of nonnegative matrices. In this case, however, it is known that the properties (i) $\div$ (iii) of Theorem 2.3 admit the following generalization: the spectral radius is always a nonnegative eigenvalue, and its corresponding (left and right) eigenvectors are nonnegative, see [34, Thm 8.3.1]. The Perron-Frobenius Theorem shows that this result can be further extended, and in particular that matrices that are nonnegative and irreducible satisfy the first three properties of Theorem 2.3.

Theorem 2.4 (Perron-Frobenius, 8.4.4 in [34]). Let $A \in \mathbb{R}^{n \times n}(n \geq 2)$ be nonnegative and irreducible. Then

(i) $\rho(A)>0$ is an algebraically simple eigenvalue of $A$; 
(ii) there is a unique real vector $\chi \in \mathbb{R}^{n}$ such that $A \chi=\rho(A) \chi$, and $\chi>0$;

(iii) there is a unique real vector $\xi \in \mathbb{R}^{n}$ such that $\xi^{T} A=\rho(A) \xi^{T}$, and $\xi>0$.

Remark 2.1. When $A$ is a positive, or a nonnegative and irreducible, matrix (as in Theorems 2.3 or 2.4, respectively), its eigenvalue $\rho(A)$ is called the Perron eigenvalue, and the corresponding left and right eigenvectors $\chi>0$ and $\xi>0$ are called the Perron eigenvectors.

\section{Characterization of matrices that possess the strong Perron-Frobenius property} An important difference between positive and nonnegative and irreducible matrices, is that for the latter the Perron eigenvalue may not be the unique eigenvalue with largest modulus.

If a nonnegative matrix $A$ is irreducible and $\rho(A)>\lambda(A)$ for all $\lambda(A) \in \Lambda(A)$, then it is called primitive. The class of nonnegative and primitive matrices is particularly relevant due to their asymptotic properties: indeed, if $A \geq 0$ is primitive, then $\lim _{k \rightarrow \infty}\left(\rho(A)^{-1} A\right)^{k}=\chi \xi^{T}$, where $\chi, \xi$ are the right and left Perron eigenvectors, respectively. Essentially, Perron's Theorem can be generalized to the class of nonnegative and primitive matrices.

Recently (see [38]), it has been shown that Perron's Theorem generalizes also to the class of eventually positive matrices, which are matrices that may have negative or zero elements, but become and stay positive after a certain power. To adequately state this result (see Theorem 2.5), the following two definitions are needed. A matrix $A \in \mathbb{R}^{n \times n}$ is called eventually positive (denoted $A \stackrel{\vee}{>} 0$ ) if there exists an integer $k_{0} \in \mathbb{N}_{+}$such that $A^{k}>0$ for all $k \geq k_{0}$. A matrix $A$ possesses the strong Perron-Frobenius property (denoted $A \in \mathcal{P} \mathcal{F}$ ) if its spectral radius is a simple positive eigenvalue and no other eigenvalue has the same modulus, and the corresponding right eigenvector is positive.

Theorem 2.5 (2.2 in [38]). Let $A \in \mathbb{R}^{n \times n}$. Then the following statements are equivalent:

(i) $A \in \mathcal{P} \mathcal{F}$ and $A^{T} \in \mathcal{P} \mathcal{F}$;

(ii) $A \stackrel{\vee}{>} 0$;

(iii) $A^{T} \stackrel{\vee}{>} 0$.

Remark 2.2. Observe that if $A>0$ or, if $A \geq 0$ and primitive, then $A \stackrel{\vee}{>}$.

Characterization of Metzler matrices and $\mathbf{M}$-matrices Finally, this section concludes by introducing Metzler matrices and M-matrices, for which interesting properties can be derived using Perron-Frobenius theory. These notions will turn useful in the next chapters: for instance, Chapter 3 will show that Metzler matrices have a close relationship with cooperative systems, while the properties of M-matrices will be used in Chapter 4 to examine the structure of Laplacian matrices.

A matrix $A \in \mathbb{R}^{n \times n}$ is called a Metzler matrix if it can be written as $A=B-s I$, where $B \geq 0$ and $s \in \mathbb{R}$, i.e., it if all its off-diagonal elements are nonnegative. The following lemma follows from the Perron-Frobenius Theorem. 
Lemma 2.2. Let $A=B-s I \in \mathbb{R}^{n \times n}$ be an irreducible Metzler matrix, i.e., $B \geq 0$ is irreducible and $s \in \mathbb{R}$. Then

(i) $\mu(A)$ is a simple eigenvalue of $A$, and $\mu(A)=\rho(B)-s$;

(ii) there is a unique real vector $\chi>0$ such that $A \chi=\mu(A) \chi$;

(iii) there is a unique real vector $\xi>0$ such that $\xi^{T} A=\xi^{T} \mu(A)$

A matrix $A$ is called an $M$-matrix if it can be written as $A=s I-B$, where $B \geq 0$ and $s \geq \rho(B)$. Perron-Frobenius theory has important consequences for the spectrum of M-matrices, as the following lemma shows.

Lemma 2.3. Let $A=s I-B \in \mathbb{R}^{n \times n}$ be an irreducible $M$-matrix, i.e, $B \geq 0$ is irreducible and $s \geq \rho(B)$. Then

- $\lambda_{1}(A)$ is real and simple, and $\lambda_{1}(A)=s-\rho(B) \geq 0$;

- there is a unique real vector $\chi>0$ such that $A \chi=\lambda_{1}(A) \chi$;

- there is a unique real vector $\xi>0$ such that $\xi^{T} A=\lambda_{1}(A) \xi^{T}$.

Therefore, a matrix $A$ is an M-matrix if and only if $-A$ is Metzler and all its eigenvalues have nonnegative real part. If $s=\rho(B)$ then $A$ is called a singular M-matrix. The properties of M-matrices have been extensively studied, see e.g. [39]. A noteworthy property is that the inverse of a (nonsingular) M-matrix is nonnegative. 



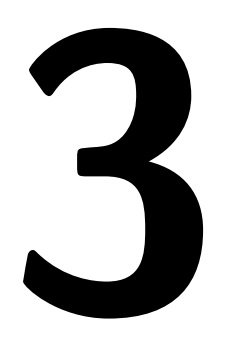

\section{Nonlinear systems}

This chapter provides background theory on nonlinear systems and on some of the tools (e.g., monotonicity, bifurcation theory) on which the analysis presented in part II relies on.

Differently from linear systems, new phenomena arise when nonlinear dynamics is involved, such as e.g., multiple isolated equilibrium points, bifurcations, and limit cycles, see [40]. Investigating the behavior of a nonlinear system means to study these phenomena, in terms of existence and stability properties. An important class of nonlinear systems, whose asymptotic behavior has been extensively studied in the literature (see for instance [41-43]), is that of monotone systems, i.e., systems which generate an order preserving flow. The first section of this chapter gives a brief overview of nonlinear systems and their analysis, and introduces the class of monotone systems.

The second section of this chapter instead focuses on bifurcation theory, which studies how the qualitative behavior of a system changes under (small) variations of its parameters.

\subsection{Preliminary definitions}

Consider a nonlinear autonomous system

$$
\dot{x}(t)=f(x(t)), \quad x(0)=x_{0},
$$

where $f: \mathcal{U} \mapsto \mathbb{R}^{n}$ is a Lipschitz continuous function on an open convex subset $\mathcal{U} \subseteq \mathbb{R}^{n}$, that is, $\exists K \geq 0$ such that $\|f(x)-f(y)\| \leq K\|x-y\|$ for all $x, y \in \mathcal{U}$. Let $\varphi\left(t, x_{0}\right)$ be the solution $x(t)$ of (3.1) that satisfies $x(0)=x_{0}$; observe that existence and uniqueness of a solution of (3.1) are guaranteed by the Lipschitz condition imposed on $f$ [40, Thm 3.2]. 
A point $x^{*}$ is an equilibrium point of (3.1) if $f\left(x^{*}\right)=0$. A nontrivial solution $x(t)$ of (3.1) is periodic of period $T$ if there exists a $T>0$ such that $x(t+T)=x(t)$ for all $t>0$. A periodic orbit is the image of a periodic solution in the phase portrait. An isolated periodic orbit is called limit cycle.

Stability of an equilibrium point is characterized in Definition 3.1, and indicates whether solutions starting close to an equilibrium point will remain close to it (stable eq. point), converge to it (asymptotically stable eq. point), or get further away from it (unstable eq. point), as $t \rightarrow \infty$, see [40, Chapter 4$]^{1}$.

Definition 3.1. An equilibrium point $x^{*}$ of (3.1) is:

- stable if, for each $\varepsilon>0$, there is a $\delta>0$ such that $\left\|x(0)-x^{*}\right\|<\delta$ implies that $\left\|x(t)-x^{*}\right\|<\varepsilon$ for all $t \geq 0$;

- asymptotically stable if it is stable and $\delta$ can be chosen such that $\left\|x(0)-x^{*}\right\|<$ $\delta$ implies that $\lim _{t \rightarrow \infty} x(t)=x^{*}$;

- unstable if it is not stable.

Without loss of generality, hereafter it will be assumed that the system (3.1) has an equilibrium point in the origin $\left(x^{*}=0 \in \mathcal{U}\right)$, and all the definitions will be stated accordingly.

Lyapunov's theory provides sufficient conditions to determine the stability properties of an equilibrium point. The well-known Lyapunov's stability theorem states that if there exists a continuously differentiable Lyapunov function, i.e., a function that is positive definite in the domain and whose derivative along the trajectories of the system is negative semidefinite, then the equilibrium point is stable. Moreover, if the derivative is negative definite, then the equilibrium point is asymptotically stable.

Theorem 3.1 (4.1 in [40]). Let the origin be an equilibrium point for (3.1). Let $V: \mathcal{U} \rightarrow \mathbb{R}_{+}$be a continuously differentiable function such that

- $V(0)=0$ and $V(x)>0$ for all $x \in \mathcal{U} \backslash\{0\}$;

- $\dot{V}(x) \leq 0$ for all $x \in \mathcal{U}$.

Then the origin is stable. Moreover, if $\dot{V}(x)<0$ for all $x \in \mathcal{U} \backslash\{0\}$ then the origin is asymptotically stable.

In addition to a sufficient condition for asymptotic stability of the origin as an equilibrium point for (3.1), Lyapunov's theory can provide also an estimate (which is often, however, conservative) for its region of attraction (or, basin of attraction), defined as the set of points $x_{0}$ such that $\lim _{t \rightarrow \infty} \varphi\left(t, x_{0}\right)=0$. Intuitively, the region of attraction indicates "how big" $\delta$ in Definition 3.1 can be for the origin to remain an attractive equilibrium point. In the particular case where the region of attraction is the whole $\mathbb{R}^{n}$, the origin is called globally asymptotically stable

\footnotetext{
${ }^{1}$ Similarly, a limit cycle is stable (resp., unstable) if all the trajectories starting arbitrarily close to it will tend toward to (resp., away from) it as $t \rightarrow \infty$ [40, Chapter 8]. This section covers key results on stability of equilibrium points only. Stability of periodic orbits is not discussed, but an example of stable limit cycle will be shown in Section 3.2.
} 
(GAS). A sufficient condition for the origin to be GAS is given by the BarbashinKrasovskii theorem (see Theorem 3.2), namely, there exists a Lyapunov function which is radially unbounded.

Theorem 3.2 (4.2 in [40]). Let the origin be an equilibrium point for (3.1). Let $V: \mathbb{R}^{n} \rightarrow \mathbb{R}_{+}$be a continuously differentiable function such that

- $V(0)=0$ and $V(x)>0$ for all $x \neq 0$;

- $\lim _{\|x\| \rightarrow+\infty} V(x)=+\infty$, i.e., $V$ is radially unbounded;

- $\dot{V}(x)<0$ for all $x \neq 0$.

Then the origin is globally asymptotically stable.

An important extension of these results is given by LaSalle's Invariant Set theory; a typical application is to establish the asymptotical stability of an equilibrium point when Lyapunov's theory fails (for instance, when asymptotic stability is expected but the derivative of the candidate Lyapunov function is only negative semidefinite). LaSalle's theory is built on the concept of invariant sets for the dynamical system. A set $\mathcal{M}$ is called (positively, omitted hereafter) invariant w.r.t. (3.1) if every trajectory starting in $\mathcal{M}$ remains in $\mathcal{M}$ for all time, i.e., $x(0) \in \mathcal{M}$ implies $x(t) \in \mathcal{M}$ for all $t \geq 0$.

Theorem 3.3 (LaSalle, 4.4 in [40]). Let $\Omega \subset \mathcal{U}$ be a compact set, which is positively invariant w.r.t. (3.1). Let $V: \mathcal{U} \rightarrow \mathbb{R}$ be a continuously differentiable function such that

- $\dot{V}(x) \leq 0$ for all $x \in \Omega$;

- $\mathcal{Z}=\{x \in \Omega: \dot{V}(x)=0\}$.

Then $\varphi\left(t, x_{0}\right)$ converges to $\mathcal{M}$ for all $x_{0} \in \Omega$, where $\mathcal{M}$ is the largest invariant set in $\mathcal{Z}$.

A consequence of LaSalle's invariance principle is that the origin is an asymptotically stable equilibrium point of (3.1) if there exists a Lyapunov function $V$, and no solution (except for the trivial solution) can stay identically in the set $\mathcal{Z}=\{x \in \Omega: \dot{V}(x)=0\}[40$, Corollary 4.1].

Remark 3.1. In Theorems 3.1, 3.2 and 3.3, the hypothesis that the Lyapunov function $V$ is continuously differentiable is not essential. However, if this assumption is removed, the derivative needs to be substituted by the upper Dini derivative of $V$ in the theorems.

The following material on the upper Dini derivative is from [44]. The upper Dini derivative of a continuous function $r:(a, b) \rightarrow \mathbb{R}$, with $a, b \in \mathbb{R}$, at $t \in(a, b)$ is defined as

$$
d^{+} r(t)=\limsup _{s \rightarrow 0^{+}} \frac{r(s+t)-r(t)}{s} .
$$

If $r$ is Lipschitz on some neighborhood of $t$ then the derivative is finite. Key result is that $r$ is decreasing on $(a, b)$ if and only if $d^{+} r(t) \leq 0$ for all $t \in(a, b)$.

Now, let $V: \mathcal{U} \rightarrow \mathbb{R}$ be a continuous and locally Lipschitz function, and $x(t)$ be a solution of (3.1). The upper Dini derivative of $V(x(t))$ is defined accordingly to (3.1), $d^{+} V(x(t))=\limsup _{s \rightarrow 0^{+}} \frac{V(x(s+t))-V(x(t))}{s}$. The upper Dini derivative of $V$ along the 
vector field $f$ of (3.1), denoted $d^{+} V$ or $d_{f}^{+} V$, is given by

$$
d^{+} V(x)=\limsup _{s \rightarrow 0^{+}} \frac{V(x+s f(x))-V(x)}{s},
$$

and it holds that $\left.d^{+} V(x)\right|_{x=x^{*}}=\left.d^{+} V(x(t))\right|_{t=t^{*}}$ with $x\left(t^{*}\right)=x^{*}$. If $d^{+} V(x) \leq 0$ on $\mathcal{U}$, then $V$ is decreasing along the solutions of (3.1).

The theorems discussed so far are main results of the Lyapunov's direct method, which focuses on finding a Lyapunov function $V$. The next theorem, denoted Lyapunov's indirect method, offers an alternative way to determine the stability of an equilibrium point, by means of linearization.

Theorem 3.4 (4.7 in [40]). Let the origin be an equilibrium point for (3.1), and assume that $f$ is continuously differentiable in $\mathcal{U}$. Let $f_{x}(x):=\frac{\partial f}{\partial x}(x)$ indicate the Jacobian of (3.1) evaluated at $x$. Then the origin is:

- asymptotically stable if $f_{x}(0)$ is Hurwitz, i.e., $\operatorname{Re}[\lambda]<0$ for all $\lambda \in \Lambda\left(f_{x}(0)\right)$;

- unstable if there exists at least one eigenvalue of $f_{x}(0)$ with positive real part.

This method fails when $f_{x}(0)$ has eigenvalues with nonpositive real part and at least one eigenvalue with zero real part. In this case, more advanced tools from center manifold theory can be used to study the stability properties of the origin [40, Chapter 8]. It is out of the scope of this thesis work to detail center manifold theory, however a brief overview is given in what follows, which will be useful in Section 3.2. Assume that the origin is an equilibrium point of the system (3.1). The generalized eigenspaces of $f_{x}(0)$ relative to eigenvalues with strictly negative, zero, and strictly positive real part are denoted stable, center, and unstable generalized eigenspaces, respectively. Center manifold theory states that it is possible to define three manifolds $\mathcal{W}_{i}, i=s, c, u$, denoted the stable, center and unstable manifolds, respectively, which are invariant w.r.t. (3.1) and tangent to the generalized stable, center and unstable eigenspaces eigenspaces of $f_{x}(0)$ at 0 , respectively. When there is no unstable manifold, the stability properties of the origin as an equilibrium point of (3.1) can be investigated by studying the dynamics of (3.1) restricted to the center manifold $\mathcal{W}_{c}$, referred to as the reduced system. If the origin is an asymptotically stable (resp., unstable) equilibrium point of the reduced dynamics then it is also an asymptotically stable (resp., unstable) equilibrium point of the original (higher-order) system [40, 45].

Discrete-time systems The notions and results introduced in Section 3.1 for continuous-time systems admit a natural extension and can be restated for discretetime nonlinear systems, represented as

$$
x(k+1)=f(x(k)), \quad x(0)=x_{0} .
$$

For instance, a point $x^{*}$ is an equilibrium point of (3.2) if $f\left(x^{*}\right)=x^{*}$. Provided that the derivative of $V$ is substituted with the increment of $V$ along the trajectories, $V_{\Delta}(x):=V(f(x))-V(x)$, Theorems 3.1 and 3.2 can be used to provide sufficient 
conditions for stability and asymptotical stability, and global asymptotic stability, respectively, of an equilibrium point. The discrete-time version of Lyapunov's indirect method can be stated as follows.

Theorem 3.5. Let the origin be an equilibrium point for (3.2), and assume that $f$ is continuously differentiable in $\mathcal{U}$. Let $f_{x}(x):=\frac{\partial f}{\partial x}(x)$ indicate the Jacobian of (3.2) evaluated at $x$. Then the origin is:

- asymptotically stable if all the eigenvalues of $f_{x}(0)$ are inside the unit circle, i.e., $|\lambda|<1$ for all $\lambda \in \Lambda\left(f_{x}(0)\right)$;

- unstable if there exists at least one eigenvalue of $f_{x}(0)$ outside the unit circle, i.e., $\exists \lambda \in \Lambda\left(f_{x}(0)\right)$ such that $|\lambda|>1$.

When $f_{x}(0)$ has eigenvalues inside the unit circle and at least one eigenvalue on the unit circle, tools from center manifold theory (as in the continuous-time case) can be used to study the stability properties of the origin as an equilibrium point of the system (3.2), see for instance [46].

\subsubsection{Cooperativity and Monotonicity}

An interesting class of nonlinear systems is that of monotone systems, whose characteristic asymptotic properties have motivated their comprehensive analysis in the literature.

Consider a nonlinear system, described as (3.1). To introduce the concept of monotonicity, the notions of orthant of $\mathbb{R}^{n}$ and of partial ordering generated by an orthant are needed. Let $S \in \mathbb{R}^{n \times n}$ be a signature matrix, i.e., $S=\operatorname{diag}\left\{s_{1}, \ldots, s_{n}\right\}$ with $s_{i}= \pm 1$ for all $i=1, \ldots, n$, and let $S \mathbb{R}^{n}$ indicate an orthant of $\mathbb{R}^{n}, S \mathbb{R}^{n}=$ $\left\{x \in \mathbb{R}^{n}: s_{i} x_{i} \geq 0, i=1, \ldots, n\right\}$. Let $\leq_{S \mathbb{R}^{n}}$ indicate the partial ordering generated by $S \mathbb{R}^{n}$, i.e., $x \leq_{S \mathbb{R}^{n}} y$ if and only if $y-x \in S \mathbb{R}^{n}$.

Definition 3.2. The partial ordering $\leq_{S \mathbb{R}^{n}}$ is preserved by the solution operator $\varphi(t, \cdot)$ and the system $(3.1)$ is type $S \mathbb{R}^{n}$ monotone if whenever $x, y \in \mathcal{U}$ and $x \leq_{S \mathbb{R}^{n}}$ $y$ then $\varphi(t, x) \leq_{S \mathbb{R}^{n}} \varphi(t, y)$ for all $t \geq 0$.

Lemma 3.1 (2.1 in [42]). If $f \in C^{1}(\mathcal{U})$ where $\mathcal{U}$ is open and convex in $\mathbb{R}^{n}$ then $\varphi(t, \cdot)$ preserves the partial ordering $\leq_{S \mathbb{R}^{n}}$ for $t \geq 0$ if and only if $S \frac{\partial f}{\partial x}(x) S$ has nonnegative off-diagonal elements for every $x \in \mathcal{U}$.

As previously stated, the importance behind the analysis of monotone systems lies in their asymptotic behavior. In particular, it is known that if all the trajectories of a monotone system are bounded, then they generically converge to an equilibrium point, and that there are no attracting periodic orbits other than equilibria [43].

Cooperativity is a special case of monotonicity corresponding to $S \mathbb{R}^{n}=\mathbb{R}_{+}^{n}$, where $\mathbb{R}_{+}^{n}$ is the nonnegative orthant. The partial ordering generated by $\mathbb{R}_{+}^{n}$ is denoted $\leq$.

Definition 3.3. The partial ordering $\leq$ is preserved by the solution operator $\varphi(t, \cdot)$ and the system $(3.1)$ is cooperative if whenever $x, y \in \mathcal{U}$ and $x \leq y$ then $\varphi(t, x) \leq \varphi(t, y)$ for all $t \geq 0$. 
The next theorem provides a necessary and sufficient condition for a vector field $f$ to determine a cooperative system.

Theorem 3.6 (12.11 in [47]). The system (3.1) is cooperative if and only if it satisfies the Kamke-Muller condition:

$$
x \leq y \quad \Rightarrow \quad f_{i}(x) \leq f_{i}(y) \quad \forall i \text { such that } x_{i}=y_{i}
$$

If $f \in C^{1}(\mathcal{U})$, a necessary and sufficient condition is that $\frac{\partial f}{\partial x}(x)$ has nonnegative off-diagonal elements for every $x \in \mathcal{U}$.

Remark 3.2. Observe that in Theorem 3.6, the necessary and sufficient condition for a differentiable vector field $f$ to determine a cooperative system is that $\frac{\partial f}{\partial x}(x)$ is Metzler (in the domain). This implies that, if the Jacobian of (3.1) is Metzler, then $\mathbb{R}_{+}^{n}$ is positively invariant w.r.t. (3.1).

\subsection{Bifurcation analysis}

In this section a brief overview of bifurcation analysis for nonlinear autonomous systems is presented, both in continuous-time and discrete-time. The main idea is that when a nonlinear system is dependent on a parameter, its qualitative behavior may change as the value of the parameter varies. A bifurcation is defined as a change in the number of equilibrium points or periodic orbits, or in their stability properties, as a parameter is varied. The parameter is called bifurcation parameter, and the points at which changes occur are called bifurcation points. A visual representation of a bifurcation is given by a bifurcation diagram [40].

A bifurcation can occur only when, for a certain value of the bifurcation parameter, the Jacobian of the system at an equilibrium point has (at least) one eigenvalue on the imaginary axis (in continuous-time systems), or has (at least) one eigenvalue of the unit circle (in discrete-time systems), see Fig. 3.1, which implies that the equilibrium point has a center manifold ${ }^{2}$; for simplicity, this thesis work considers only the class of steady-state bifurcations for which the center manifold has dimension one.

From center manifold theory, in order to investigate the qualitative behavior of a nonlinear (in general, $n$-dimensional) system near a bifurcation point it is sufficient to study the center manifold (scalar) dynamics. A key concept of bifurcation theory is that each steady-state bifurcation can be represented by a canonical scalar system, called normal form, and that every problem can be reduced to a specific normal form exhibiting the same qualitative behavior under variation of a parameter.

This chapter provides a brief introduction on the normal forms of (steady-state) bifurcations that are of interest for this thesis work, and, later, on the procedure to reduce general (higher-dimensional) problems to these canonical forms. It focuses specifically on systems that have an odd symmetry, denoted $\mathbf{Z}_{2}$-equivariant

\footnotetext{
${ }^{2}$ In this case, the equilibrium point is called nonhyperbolic [45, 46]. However, this notation is not used in the papers of Part II.
} 


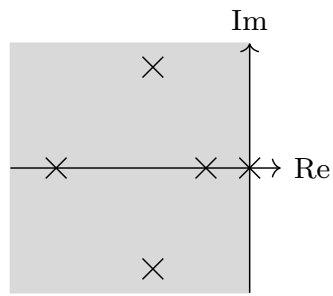

(a)

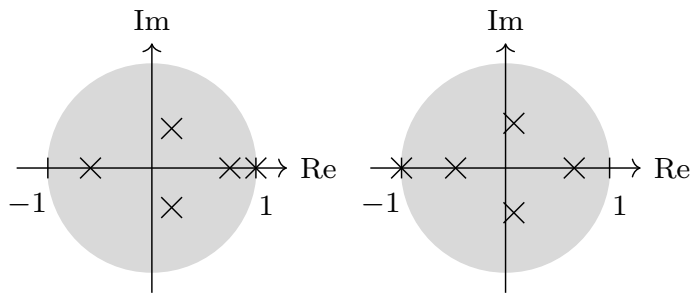

(b)

Figure 3.1: Necessary condition for occurrence of a bifurcation at an equilibrium point. (a): The Jacobian at the equilibrium point has eigenvalues with zero real part (continuous-time systems). (b): The Jacobian at the equilibrium point has eigenvalues on the unit circle (discrete-time systems). Legend: crosses indicate eigenvalues of the Jacobian at an equilibrium point.

systems $[48,49]$, which exhibit a specific type of steady-state bifurcation, denoted pitchfork bifurcation.

Since the (stability) characterization of equilibrium points for continuous-time and discrete-time systems is different, these cases will be treated separately. The material presented in this chapter is inspired mostly by $[45,46,48]$.

\subsubsection{Continuous-time systems: pitchfork bifurcation}

Consider the nonlinear system

$$
\dot{x}=f(x, \pi), \quad x(0)=x_{0},
$$

where $x \in \mathbb{R}^{n}, \pi \in \mathbb{R}$ is a scalar parameter, and $f: \mathbb{R}^{n} \rightarrow \mathbb{R}^{n}$ is infinitely differentiable everywhere. Let $f_{x}(x, \pi):=\frac{\partial f}{\partial x}(x, \pi)$ indicate the Jacobian of (3.3) evaluated at $(x, \pi)$. A point $x^{*}$ is an equilibrium point of (3.3) at $\pi=\pi^{*}$ if $f\left(x^{*}, \pi^{*}\right)=0$.

Definition 3.4. The parameter $\pi$ is called bifurcation parameter, and the set of $(x, \pi)$ satisfying $f(x, \pi)=0$ bifurcation diagram. A point $\left(x^{*}, \pi^{*}\right)$ is called bifurcation point if the number of solutions of $f(x, \pi)=0$ changes as $\pi$ varies in a neighborhood of $\pi^{*}$.

Necessary conditions for a bifurcation to occur at a point $\left(x^{*}, \pi^{*}\right)$ are that $f\left(x^{*}, \pi^{*}\right)=0$, and $f_{x}\left(x^{*}, \pi^{*}\right)$ has an eigenvalue on the imaginary axis. Without loss of generality, in what follows it is assumed that $\left(x^{*}, \pi^{*}\right)=(0,0)$, and that the zero eigenvalue of $f_{x}(0,0)$ is real and simple $e^{3}$.

This section focuses on a specific type of (steady-state) bifurcation, called pitchfork bifurcation, characterized by the fact that at the bifurcation point the number of solutions of $f(x, \pi)=0$ jumps from one to three. This type of bifurcation is ubiquitous of systems that have odd symmetry, i.e., systems for which $f(x, \pi)=-f(-x, \pi)$ for all $x \in \mathbb{R}^{n}$.

\footnotetext{
${ }^{3}$ The case in which $f_{x}(0,0)$ has complex conjugate eigenvalues on the imaginary axis is not considered.
} 


\section{Normal form of a pitchfork bifurcation}

The normal form of a pitchfork bifurcation is described by the scalar nonlinear system

$$
\dot{x}=\pi x-x^{3}, \quad x \in \mathbb{R}, \pi \in \mathbb{R} .
$$

Let $g(x, \pi)=-x^{3}+\pi x$ and $g_{x}(x, \pi)=-3 x^{2}+\pi$. Observe that the system $(3.4)$ has odd symmetry since $g$ is odd in $x$.

The origin is an equilibrium point of the system (3.4) for all values of $\pi \in \mathbb{R}$. It is globally asymptotically stable when $\pi \leq 0$, and unstable when $\pi>0$. When $\pi>0$ the system admits two new equilibrium points in $\pm \sqrt{\pi}$, which are locally asymptotically stable since $g_{x}( \pm \sqrt{\pi}, \pi)<0$ for all $\pi>0$. This means that the system (3.4) undergoes a pitchfork bifurcation at $\pi=0$, and the corresponding bifurcation diagram is depicted in Figure $3.2^{4}$.

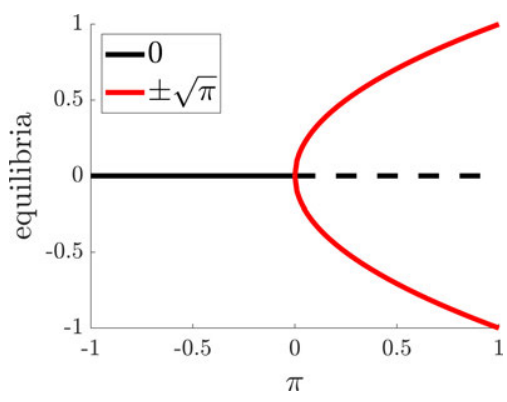

Figure 3.2: Bifurcation diagram of a pitchfork bifurcation, whose normal form is shown in (3.4). Solid lines indicate stable equilibrium points, while a dashed line unstable equilibrium points.

\section{How to recognize a pitchfork bifurcation}

In the previous section, the normal form of a pitchfork bifurcation has been introduced. In general if a bifurcation problem, represented by a nonlinear system

$$
\dot{x}=g(x, \pi), \quad x \in \mathbb{R}, \pi \in \mathbb{R},
$$

at a point $(x, \pi)=(0,0)$ satisfies the conditions

$$
g=g_{x}=g_{x x}=g_{\pi}=0, \quad g_{x x x}<0, \quad g_{\pi x}>0,
$$

then it is said to be equivalent to the normal form (3.4), and (3.6) is said to solve the recognition problem for the pitchfork bifurcation ${ }^{5}$. Intuitively, this means that there exists an adequate change of coordinates that transforms the problem

\footnotetext{
${ }^{4}$ Figure 3.2 shows a supercritical pitchfork bifurcation. A subcritical pitchfork bifurcation instead is characterized by the number of equilibria jumping from 3 to 1 , and is described by the normal form $g(x, \pi)=\pi x+x^{3}=0$. This thesis work focuses only on the supercritical pitchfork bifurcation, therefore the term "supercritical" will be dropped from now on.

${ }^{5}$ The subscripts in (3.6) indicate partial derivatives.
} 
$g(x, \pi)=0$ into the standard form of a pitchfork, $-x^{3}+\pi x=0$, see $[48, \S 1]$ for details. Therefore, the number of solutions of $g(x, \pi)=0$ jumps from one to three as $\pi$ crosses 0 .

Similarly, every nonlinear model that exhibits a pitchfork bifurcation behavior can be reduced to a scalar differential equation $\dot{x}=g(x, \pi)$ that satisfies $(3.6)$. A method to obtain such scalar equation from a general $n$-dimensional system is the Lyapunov-Schmidt reduction, explained in [48, §3] and used in Papers B and C.

\subsubsection{Discrete-time systems: pitchfork and period-doubling bifurcations}

Consider the nonlinear system

$$
x(k+1)=f(x(k), \pi), \quad x(0)=x_{0},
$$

where $x \in \mathbb{R}^{n}, \pi \in \mathbb{R}$ is a scalar parameter, and $f: \mathbb{R}^{n} \rightarrow \mathbb{R}^{n}$ is infinitely differentiable everywhere. Let $f_{x}(x, \pi):=\frac{\partial f}{\partial x}(x, \pi)$ indicate the Jacobian of (3.7) evaluated at $(x, \pi)$. A point $x^{*}$ is an equilibrium point of $(3.7)$ at $\pi=\pi^{*}$ if $f\left(x^{*}, \pi^{*}\right)=x^{*}$.

Definition 3.5. The parameter $\pi$ is called bifurcation parameter, and the set of $(x, \pi)$ satisfying $f(x, \pi)=x$ bifurcation diagram. A point $\left(x^{*}, \pi^{*}\right)$ is called bifurcation point if the number of solutions of $f(x, \pi)=x$ changes as $\pi$ varies in a neighborhood of $\pi^{*}$.

Necessary conditions for a bifurcation to occur at a point $\left(x^{*}, \pi^{*}\right)$ are that $f\left(x^{*}, \pi^{*}\right)=x^{*}$, and $f_{x}\left(x^{*}, \pi^{*}\right)$ has an eigenvalue on the unit circle. Again, without loss of generality, in what follows it is assumed that $\left(x^{*}, \pi^{*}\right)=(0,0)$, and that the eigenvalue of $f_{x}(0,0)$ on the unit circle is real and simple, that is, $\exists \lambda \in \Lambda\left(f_{x}(0,0)\right)$ such that either $\lambda=+1$ or $\lambda=-1$.

The type of bifurcation the system (3.7) undergoes at $(0,0)$ depends on the sign of the eigenvalue $\lambda$. The next paragraphs introduce the normal forms of a pitchfork bifurcation ( $\lambda=1$, assuming that the system has odd symmetry), and of a period-doubling bifurcation $(\lambda=-1)$.

\section{Normal form of a pitchfork bifurcation}

The normal form of a pitchfork bifurcation is described by the scalar nonlinear system

$$
x(k+1)=(1+\pi) x(k)-x(k)^{3}, \quad x \in \mathbb{R}, \pi \in \mathbb{R} .
$$

Let $g(x, \pi)=(1+\pi) x-x^{3}$ and $g_{x}(x, \pi)=(1+\pi)-3 x^{2}$. The system (3.8) has odd symmetry since $g$ is odd in $x$.

The analysis is similar to the continuous-time case. The origin is an equilibrium point of the system (3.4) for all values of $\pi \in \mathbb{R}$, and it is stable when $\pi \leq 0$ (and $|\pi|$ small), and unstable when $\pi>0$. When $\pi>0$ the system admits two new equilibrium points in $\pm \sqrt{\pi}$, which are locally asymptotically stable since $g_{x}( \pm \sqrt{\pi}, \pi)<1$ for all $\pi>0$ (and $\pi$ small).

This means that the system (3.4) undergoes a pitchfork bifurcation at $\pi=0$, and the corresponding bifurcation diagram is the same as Fig. 3.2. 


\section{Normal form of a period-doubling bifurcation}

The normal form of a period-doubling bifurcation is described by the scalar nonlinear system

$$
x(k+1)=-(1+\pi) x(k)+x(k)^{3}, \quad x \in \mathbb{R}, \pi \in \mathbb{R} .
$$

Let $g(x, \pi)=-(1+\pi) x+x^{3}$ and $g_{x}(x, \pi)=-(1+\pi)+3 x^{2}$. The origin is an equilibrium point of the system (3.9) for all values of $\pi \in \mathbb{R}$. It is asymptotically stable when $\pi<0$ (and $|\pi|$ small), and unstable when $\pi>0$.

For values of $\pi$ near 0 the system (3.9) does not admit any other equilibrium point. Instead, as the name suggests, the characteristic of a period-doubling bifurcation is that it produces an oscillation of period 2. To understand, one needs to consider the second iterate of the system (3.9),

$$
x(k+2)=g^{2}(x(k), \pi)
$$

described by

$$
g^{2}(x, \pi):=g(g(x, \pi), \pi)=(1+\pi)^{2} x-(1+\pi)\left(2+2 \pi+\pi^{2}\right) x^{3}+O\left(x^{5}\right),
$$

and observe that a nontrivial equilibrium point of (3.10) corresponds to a periodic oscillation of (3.9) of period 2 .

The bifurcation analysis of the system (3.10), see Fig. 3.3, shows that (3.10) undergoes a pitchfork bifurcation from the origin at $\pi=0$, which implies that (3.9) admits a stable limit cycle of period 2 when $\pi$ crosses 0 .

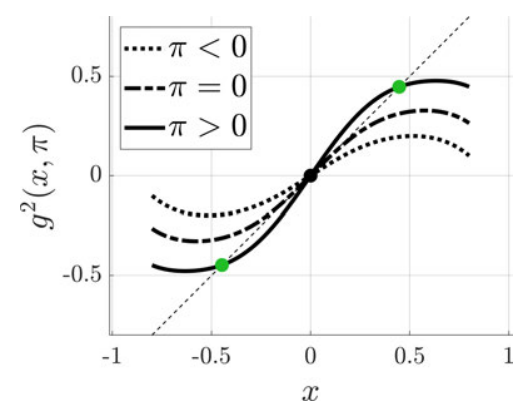

(a)

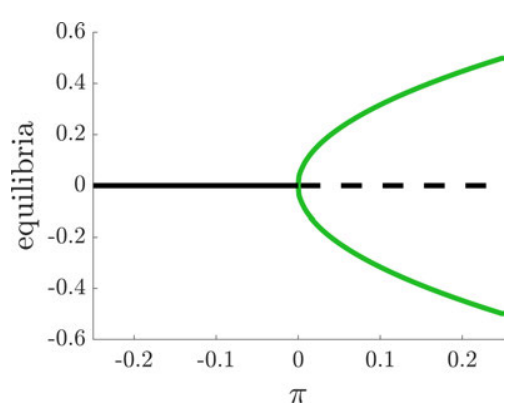

(b)

Figure 3.3: Analysis of the second iterate (3.10) near $(0,0)$. (a): $g^{2}(x, \pi)$ near $(0,0)$ for different values of $\pi$. (b): Bifurcation diagram of (3.10).

\section{How to recognize a pitchfork or a period-doubling bifurcation}

In the previous section, the normal forms of a pitchfork and a period-doubling bifurcation have been introduced. In general if a bifurcation problem, represented by a scalar nonlinear system

$$
x(k+1)=g(x(k), \pi), \quad x \in \mathbb{R}, \pi \in \mathbb{R},
$$


at a point $(x, \pi)=(0,0)$ satisfies the conditions

$$
g=0, \quad g_{x}=1, \quad g_{x x}=0, \quad g_{\pi}=0, \quad g_{x x x}<0, \quad g_{\pi x}>0,
$$

then it is equivalent to the normal form of a pitchfork bifurcation (3.8). If, instead, it satisfies the conditions

$$
g=0, \quad g_{x}=-1, \quad g_{x x}=0, \quad g_{\pi}=0, \quad g_{x x x}>0, \quad g_{\pi x}<0,
$$

then it is equivalent to the normal form of a period-doubling bifurcation (3.9).

In $n$-dimensional systems (3.7), the bifurcation analysis reduces to that of the dynamics along the center manifold of the equilibrium point. The details can be found in [46, Chapter 5]. 



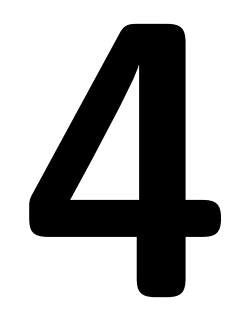

\section{Theory of graphs and social networks}

Graphs are the natural mathematical structure to model networks of interacting agents. For instance, a social network can be modeled as a graph where the nodes represent individuals, and the edges the social interactions between them [10]. The general aim of this chapter is to introduce key notions and results from graph theory, i.e., the study of networks structure, and the interpretation will be given in terms of social networks. In particular, the second part of this chapter is dedicated to the theory of signed graphs, which are used to model networks where the interactions between the agents are not restricted to be friendly or cooperative, but can be hostile or antagonistic.

The main references for the material of this chapter are [50-52] and [14, 16, 53, 54 ], for graph and signed graph theory, respectively. In the rest of this thesis work the terms graphs and networks will be used interchangeably.

\subsection{Graphs}

A graph $\mathcal{G}$ is defined as a triple $\mathcal{G}=(\mathcal{V}, \mathcal{E}, A)$ where $\mathcal{V}=\{1, \ldots, n\}$ is a finite set of nodes, $\mathcal{E} \subseteq \mathcal{V} \times \mathcal{V}$ is a set of edges, and $A=\left[a_{i j}\right] \in \mathbb{R}_{+}^{n \times n}$ is the adjacency matrix of $\mathcal{G}$, i.e., $a_{i j}>0$ if and only if $(j, i) \in \mathcal{E}$. A graph is unweighted if $a_{i j} \in\{0,1\}$ for all $i, j \in \mathcal{V}$, and it is weighted otherwise.

A self-loop is an edge from a node to itself.

A graph $\mathcal{G}$ is called undirected if $\mathcal{E}$ is a set of unordered edges, i.e., if $(i, j) \in \mathcal{E}$ implies that $(j, i) \in \mathcal{E}$, and it is called directed (or, a digraph) if $\mathcal{E}$ is a set of ordered edges. In this case, $(i, j) \in \mathcal{E}$ denotes an edge from $i$ to $j$.

A (directed) path in a graph is an ordered sequence of nodes such that any pair of consecutive nodes in the sequence is a (directed) edge of the graph. It is simple if all nodes in the (directed) path are distinct. A graph is (strongly) connected if there is a (directed) path between any two distinct nodes. A cycle is a simple 


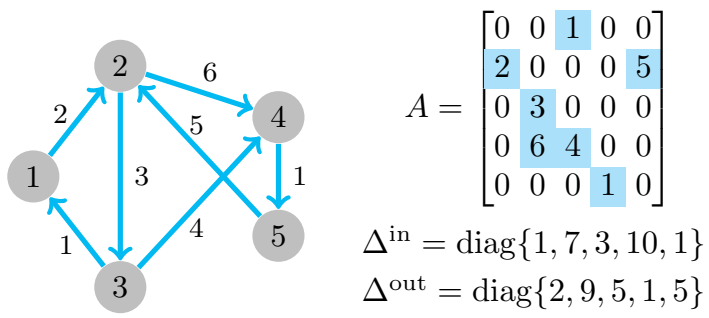

(a)

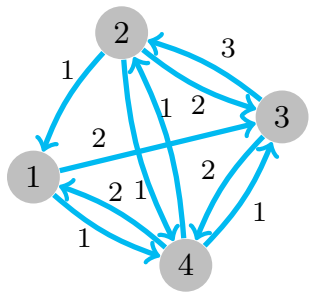

$$
\begin{gathered}
A=\left[\begin{array}{llll}
0 & 1 & 0 & 2 \\
0 & 0 & 3 & 1 \\
2 & 2 & 0 & 1 \\
1 & 1 & 2 & 0
\end{array}\right] \\
\Delta^{\text {in }}=\Delta^{\text {out }}=\operatorname{diag}\{3,4,5,4\}
\end{gathered}
$$

(b)

Figure 4.1: Examples of simple, weighted graphs. (a): Strongly connected digraph with $\operatorname{card}(\mathcal{V})=5$ nodes and 7 directed edges, and corresponding adjacency, in-degree and out-degree matrices. (b): Weight balanced graph with $\operatorname{card}(\mathcal{V})=4$ nodes and 10 directed edges, and corresponding adjacency, in-degree and out-degree matrices.

(directed) path that starts and ends at the same node.

A node $j$ is a in-neighbor of $i$ (or, $j$ is adjacent to $i$ ) if $(j, i) \in \mathcal{E}$, and is a outneighbor of $i$ if $(i, j) \in \mathcal{E}$, and the in-neighborhood $\mathcal{N}_{i}^{\text {in }}$ and out-neighborhood $\mathcal{N}_{i}^{\text {out }}$ of a node $i$ indicate the set of all in- and out-neighbors of $i$, respectively. A node is called isolated if its in-neighborhood and out-neighborhood are both empty sets. A graph is complete if every pair of nodes is adjacent, i.e., $\mathcal{N}_{i}^{\text {in }}=\mathcal{N}_{i}^{\text {out }}=\mathcal{V} \backslash\{i\}$ for all $i \in \mathcal{V}$.

The in-degree and out-degree of a node $i \in \mathcal{V}$ are defined by $\delta_{i}^{\text {in }}=\sum_{j=1}^{n} a_{i j}$ and $\delta_{i}^{\text {out }}=\sum_{j=1}^{n} a_{j i}$, respectively. A node is called isolated if its in-degree and out-degree are both zero (i.e., $\delta_{i}^{\text {in }}=\delta_{i}^{\text {out }}=0$ ). A graph is called weight-balanced if in-degree and out-degree coincide for each node. If a graph is undirected then it is also weight-balanced and in this case the superscripts "in" and "out" are dropped $\left(\delta_{i}^{\text {in }}=\delta_{i}^{\text {out }}=: \delta_{i}\right.$ for all $i$ ). The in-degree matrix and out-degree matrix of a graph are defined by $\Delta^{\text {in }}=\operatorname{diag}\left\{\delta_{1}^{\text {in }}, \ldots, \delta_{n}^{\text {in }}\right\}$ and $\Delta^{\text {out }}=\operatorname{diag}\left\{\delta_{1}^{\text {out }}, \ldots, \delta_{n}^{\text {out }}\right\}$, respectively. This thesis work mostly deals with the notion of in-degrees and, when there is no ambiguity, the notation $\delta_{i}$ and $\Delta$ is used to indicate the in-degree of a node $i$ and the in-degree matrix, respectively.

These concepts are illustrated in the examples of Figure 4.1.

Remark 4.1. The adjacency matrix of a graph $\mathcal{G}=(\mathcal{V}, \mathcal{E}, A)$ has the following properties:

- $\mathcal{G}$ is without self-loops if and only if $A$ has zero diagonal elements;

- $\mathcal{G}$ has an isolated node $i$ if and only if the $i$-th row and $i$-th column of $A$ are zero;

- $\mathcal{G}$ is undirected if and only if $A=A^{T}$; 
- $\mathcal{G}$ is connected/strongly connected if and only if $A$ is irreducible, i.e., if there does not exist a permutation matrix $P$ such that $P^{T} A P$ is block triangular;

- $\mathcal{G}$ is weight-balanced if and only if $A \mathbb{1}=A^{T} \mathbb{1}$, where $\mathbb{1}$ is the vector of all 1 .

Remark 4.2. This thesis work considers graphs that are (strongly) connected and without self-loops.

\subsubsection{Laplacian of a graph and its properties}

This section introduces the definition of Laplacian matrix of a graph. The definitions and lemmas are stated for the general case of directed graphs; however, they hold also for undirected graphs.

The Laplacian of a graph $\mathcal{G}=(\mathcal{V}, \mathcal{E}, A)$, with $\operatorname{card}(\mathcal{V})=n$, is the matrix $L \in$ $\mathbb{R}^{n \times n}$ defined by

$$
[L]_{i j}= \begin{cases}\sum_{k=1}^{n} a_{i k}, & j=i \\ -a_{i j}, & j \neq i .\end{cases}
$$

In compact form, $L=\Delta^{\text {in }}-A$.

The following lemma is a collection of fundamental and well-known properties of the Laplacian matrix, see for instance [51, 52, 55-57]. Intuitively, (i) $\div$ (vi) follow directly from the definition of Laplacian, the Geršgorin's Theorem, and the Perron-Frobenius Theorem, and (vii) is shown in [57, Theorem 2].

Lemma 4.1. The Laplacian $L$ of a digraph $\mathcal{G}=(\mathcal{V}, \mathcal{E}, A)$, with $\operatorname{card}(\mathcal{V})=n$, has the following properties:

(i) $L \mathbb{1}=0$, i.e., 0 is always an eigenvalue of $L$ with right eigenvector $\mathbb{1}$;

(ii) all the eigenvalues of $L$ have nonnegative real part, i.e., $\operatorname{Re}[\lambda(L)] \geq 0$ for all $\lambda(L) \in \Lambda(L)$

(iii) $L$ is a singular $M$-matrix.

Moreover, if the digraph $\mathcal{G}$ is strongly connected, then:

(iv) $L$ is a singular and irreducible M-matrix;

(v) the zero eigenvalue of $L$ is simple, $\operatorname{rank}(L)=n-1$ and $\operatorname{ker}(L)=\operatorname{span}\{\mathbb{1}\}$;

(vi) the left eigenvector of $L$ associated with the zero eigenvalue is positive, i.e., $\xi>0$ with $L^{T} \xi=0$ and $\operatorname{ker}\left(L^{T}\right)=\operatorname{span}\{\xi\} ;$

(vii) $\xi>0$ is the unique vector (up to a scaling factor) such that $\Xi L+L^{T} \Xi$ is positive semidefinite, with $\Xi:=\operatorname{diag}\{\xi\}$, and $\operatorname{rank}\left(\Xi L+L^{T} \Xi\right)=n-1$.

Something more can be added when a digraph is not only strongly connected but also weight-balanced, as shown for instance in the recent work [57].

Lemma 4.2. Let $\mathcal{G}=(\mathcal{V}, \mathcal{E}, A)$, with $\operatorname{card}(\mathcal{V})=n$, be a strongly connected digraph with Laplacian $L$. The following statements are equivalent:

(i) $\mathcal{G}$ is weight-balanced;

(ii) $L^{T} \mathbb{1}=0$ and, therefore, $\operatorname{ker}(L)=\operatorname{ker}\left(L^{T}\right)=\operatorname{span}\{\mathbb{1}\}$; 
(iii) the symmetric part of $L, L_{s}:=\frac{L+L^{T}}{2}$, is positive semidefinite and $\operatorname{rank}\left(L_{s}\right)=$ $n-1$.

When a digraph $\mathcal{G}$ is strongly connected then it does not have isolated nodes, which means that the in-degree matrix $\Delta^{\text {in }}$ is positive definite. Therefore, its inverse $\left(\Delta^{\mathrm{in}}\right)^{-1}$ is well-defined and positive definite. The normalized Laplacian of a strongly connected digraph $\mathcal{G}=(\mathcal{V}, \mathcal{E}, A)$ is the matrix $\mathcal{L} \in \mathbb{R}^{n \times n}$ defined by

$$
[\mathcal{L}]_{i j}= \begin{cases}1, & j=i \\ -\frac{a_{i j}}{\sum_{k=1}^{n} a_{i k}}, & j \neq i .\end{cases}
$$

In compact form, $\mathcal{L}=I-\left(\Delta^{\text {in }}\right)^{-1} A$. The normalized Laplacian $L$ is a Laplacian matrix, therefore it satisfies the same properties of $L$ listed in Lemma 4.1. For completeness, its key properties are summarized in the following lemma: in this case, a bound for the eigenvalues of $\mathcal{L}$ can be provided using the Geršgorin's Theorem.

Lemma 4.3. The normalized Laplacian $\mathcal{L}$ of a strongly connected digraph $\mathcal{G}=$ $(\mathcal{V}, \mathcal{E}, A)$, with $\operatorname{card}(\mathcal{V})=n$, has the following properties:

(i) $\mathcal{L} \mathbb{1}=0$, i.e., 0 is always an eigenvalue of $\mathcal{L}$ with right eigenvector $\mathbb{1}$;

(ii) the zero eigenvalue of $\mathcal{L}$ is simple, $\operatorname{rank}(\mathcal{L})=n-1$ and $\operatorname{ker}(\mathcal{L})=\operatorname{span}\{\mathbb{1}\}$;

(iii) every eigenvalue of $\mathcal{L}$ lies in the closed bounded disk of center 1 and radius 1 (i.e., $|\lambda-1| \leq 1$ for all $\lambda \in \Lambda(\mathcal{L})$ );

(iv) $\mathcal{L}$ is a singular and irreducible $M$-matrix.

\subsection{Signed graphs}

A signed graph $\mathcal{G}=(\mathcal{V}, \mathcal{E}, A)$ is a graph where each edge in $\mathcal{E}$ is associated with a positive or negative sign. The corresponding adjacency matrix is a signed matrix, i.e., $\operatorname{sign}\left(a_{i j}\right)= \pm 1$ for all $(j, i) \in \mathcal{E}$, see Fig. 4.2a. A signed graph is unweighted if $a_{i j} \in\{-1,0,1\}$ for all $i, j \in \mathcal{V}$, and it is weighted otherwise. In this setting the graphs described in Section 4.1, i.e., graphs whose edges have all positive weights or, equivalently, whose adjacency matrix is nonnegative, are also denoted unsigned graphs.

Except for the notions of in-degrees and out-degrees, all the definitions introduced in Section 4.1 hold also for graphs that are signed. The notions of signed (in- and out-)degrees will be discussed in Remark 4.3 of Section 4.2.1.

\subsubsection{Signed Laplacian matrices}

In the literature of signed graphs different definitions of signed Laplacian are proposed, see [16, 54]. Using the notation introduced in [54], the opposing signed Laplacian $L^{o}$ is defined as

$$
\left[L^{o}\right]_{i j}= \begin{cases}\sum_{k=1}^{n}\left|a_{i k}\right|, & j=i \\ -a_{i j}, & j \neq i .\end{cases}
$$


while the repelling signed Laplacian $L^{r}$ is defined as

$$
\left[L^{r}\right]_{i j}= \begin{cases}\sum_{k=1}^{n} a_{i k}, & j=i \\ -a_{i j}, & j \neq i,\end{cases}
$$

When it is clear from context, the superscripts " $o$ " and " $r$ " will be dropped. ${ }^{1}$

Compared with the Laplacian of an unsigned graph introduced in Section 4.1.1, here denoted unsigned Laplacian, the signed Laplacian matrices may or may not satisfy the properties listed in Lemma 4.1, as summarized and illustrated in Table 4.1. Counterexamples, see for instance Figure 4.2, can be found to show that a certain property does not hold in the signed case.

It is possible to define a signed Laplacian matrix, i.e., a matrix that induces a simple weighted signed digraph.

Definition 4.1. A matrix $L \in \mathbb{R}^{n \times n}(n \geq 2)$ is a:

- opposing signed Laplacian matrix if

(i) $L$ has nonnegative diagonal elements;

(ii) $L$ is diagonally equipotent.

- repelling signed Laplacian matrix if

(i) $L \mathbb{1}=0$.

A signed Laplacian matrix $L$ (repelling or opposing) induces a simple weighted signed digraph $\mathcal{G}=(\mathcal{V}, \mathcal{E}, A)$ with $\operatorname{card}(\mathcal{V})=n$, where $(i, j) \in \mathcal{E}$ if and only if $[L]_{j i} \neq 0$, and $[A]_{i j}=-[L]_{i j}$ if $j \neq i$ or $[A]_{i j}=0$ otherwise.

Remark 4.3. As for the notion of signed Laplacian, in this thesis work two alternative definitions for the signed in-degrees and out-degrees of a signed graph $\mathcal{G}$ are proposed. The in-degree and out-degree of a node $i \in \mathcal{V}$ are defined either by

$$
\delta_{i}^{\text {in }}=\sum_{j=1}^{n}\left|a_{i j}\right|, \quad \delta_{i}^{\text {out }}=\sum_{j=1}^{n}\left|a_{j i}\right|,
$$

or

$$
\delta_{i}^{\text {in }}=\sum_{j=1}^{n} a_{i j}, \quad \delta_{i}^{\text {out }}=\sum_{j=1}^{n} a_{j i}
$$

in the context of the opposing or repelling signed Laplacian, respectively. Therefore, the signed Laplacian of a graph, given by (4.3) or (4.4), can be written in compact form as $L=\Delta^{\text {in }}-A$, where $\Delta^{\text {in }}$ is the signed in-degree matrix with diagonal elements given by (4.5) or (4.6), respectively.

\footnotetext{
${ }^{1}$ To avoid confusion in the papers of part II, the following legend holds:
}

- Paper A and Paper B consider unsigned graphs, and use the notion of unsigned Laplacian (4.1) and its normalization (4.2);

- Paper C and Paper D consider signed graphs, and use the notion of opposing signed Laplacian (4.3) and its normalization (4.7), defined in Section 4.3.1;

- Paper E considers signed graphs, and uses the notion of repelling signed Laplacian (4.4). 
Notice that once a definition for the signed in-degrees and out-degrees is adopted, the notion of weight balanced signed graph is equivalent to the one introduced in Section 4.1, namely, if signed in-degree and out-degree coincide for each node.

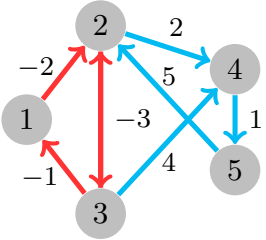

$$
A=\left[\begin{array}{ccccc}
0 & 0 & -1 & 0 & 0 \\
-2 & 0 & -3 & 0 & 5 \\
0 & -3 & 0 & 0 & 0 \\
0 & 2 & 4 & 0 & 0 \\
0 & 0 & 0 & 1 & 0
\end{array}\right]
$$

(a)

$$
L^{o}=\left[\begin{array}{ccccc}
1 & 0 & 1 & 0 & 0 \\
2 & 10 & 3 & 0 & -5 \\
0 & 3 & 3 & 0 & 0 \\
0 & -2 & -4 & 6 & 0 \\
0 & 0 & 0 & -1 & 1
\end{array}\right], \quad L^{r}=\left[\begin{array}{ccccc}
-1 & 0 & 1 & 0 & 0 \\
2 & 0 & 3 & 0 & -5 \\
0 & 3 & -3 & 0 & 0 \\
0 & -2 & -4 & 6 & 0 \\
0 & 0 & 0 & -1 & 1
\end{array}\right]
$$

(b)
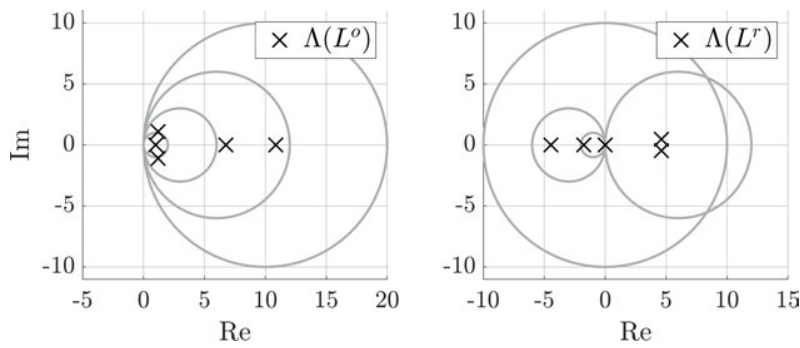

(c)

Figure 4.2: Example of a weighted signed digraph. (a): Strongly connected digraph with $\operatorname{card}(\mathcal{V})=5$ nodes and 8 directed edges, and associated adjacency matrix. A red color is used to indicate edges with negative weights, and a blue color to indicate edges with positive weights. (b): Repelling and opposing signed Laplacian. Observe that only $L^{O}$ is diagonally dominant. (c): Geršgorin's disks and eigenvalues of $L^{O}$ (left) and $L^{r}$ (right). Observe that $0 \in \Lambda\left(L^{r}\right)$ but $0 \notin \Lambda\left(L^{o}\right)$, and that while all the eigenvalues of $L^{o}$ have positive real part, $L^{r}$ has two eigenvalues with negative real

\begin{tabular}{|c|c|c|c|}
\hline$L$ & $L^{o}$ & $L^{r}$ & Property \\
\hline a & $\square$ & $\square$ & $\begin{array}{l}0 \text { is an eigenvalue } \\
\text { all the eigenvalues have nonnegative real part } \\
\text { it is diagonally dominant }\end{array}$ \\
\hline
\end{tabular}
part.

Table 4.1: Properties of the opposing and repelling signed Laplacian compared with the unsigned Laplacian. Legend: $\boldsymbol{\square}=$ the property is always satisfied, $\boldsymbol{\square}=$ the property may or may not be satisfied. 


\subsection{Social networks as signed graphs}

In the context of social networks, edges with positive weights represent friendship or cooperation, while edges with negative weights represent hostility or competition. The notion of structurally balanced network, first introduced in the field of social psychology by Heider [58] and then translated into the setting of signed graph theory by Harary and Cartwright $[15,59]$, represents a situation where, even if there are antagonistic interactions between the agents, at a network level there is no social tension. There are only two ways a social network can be structurally balanced: if, trivially, there is no antagonism between the individuals; or, if it is possible to divide the group of agents into two groups of mutual friends linked only by hostile interactions [10]. When a network is not structurally balanced, measures are proposed to characterize its distance from a structurally balanced state, see e.g., line index of balance introduced by Harary [17]. In this section these concepts are formulated in terms of (undirected) signed graphs.

\subsubsection{Structurally balanced graphs and frustration index}

Let $\mathcal{G}=(\mathcal{V}, \mathcal{E}, A)$ be a signed, undirected, and simple graph.

Definition 4.2. A signed graph is structurally balanced if there exists a partition of the node set $\mathcal{V}=\mathcal{V}_{1} \cup \mathcal{V}_{2}$, with $\mathcal{V}_{1} \cap \mathcal{V}_{2}=\emptyset$, such that every edge between $\mathcal{V}_{1}$ and $\mathcal{V}_{2}$ is negative, and every edge within $\mathcal{V}_{1}$ or $\mathcal{V}_{2}$ is positive.

Figure 4.3a illustrates a structurally balanced signed graph. An unsigned graph is a special case of structurally balanced graph, in which for instance $\mathcal{V}_{1}=\mathcal{V}$ and $\mathcal{V}_{2}=\emptyset$.

The opposing signed Laplacian $(4.3)^{2}$ has been used in the literature of signed graphs to characterize the structural balance of a signed graph $\mathcal{G}$. In compact form, $L=\Delta-A$, where $\Delta$ is the (signed) degree matrix (4.5), $\Delta:=\operatorname{diag}\left\{\delta_{1}, \ldots, \delta_{n}\right\}$ with $\delta_{i}=\sum_{j=1}^{n}\left|a_{i j}\right| \geq 0$ for all $i$. When the signed graph $\mathcal{G}$ is connected, then it does not have isolated vertices, which means that the degree matrix $\Delta$ is positive definite, and that its inverse $\Delta^{-1}$ is well-defined and positive definite. As for unsigned graphs, the normalized signed Laplacian can be defined as

$$
[\mathcal{L}]_{i j}= \begin{cases}1, & j=i \\ -\frac{a_{i j}}{\sum_{k=1}^{n}\left|a_{i k}\right|}, & j \neq i .\end{cases}
$$

which in compact form reads $\mathcal{L}=\Delta^{-1} L=I-\Delta^{-1} A$. Equivalent conditions for $\mathcal{G}$ to be structurally balanced can be formulated in terms of $\mathcal{L}$, see for instance [16].

Theorem 4.1. Let $\mathcal{G}$ be a signed connected graph $(\operatorname{card}(\mathcal{V})=n)$ with normalized signed Laplacian $\mathcal{L}$. Then $\mathcal{G}$ is structurally balanced if and only if any of the following conditions are satisfied:

(i) all the cycles in $\mathcal{G}$ are positive, i.e., they all have an even number of edges with negative weights;

\footnotetext{
${ }^{2}$ Notice that in this section the superscript "o" will be dropped, and the opposing signed Laplacian $L^{o}$ will be referred to just as signed Laplacian $L$.
} 
(ii) there exists a signature matrix $S=\operatorname{diag}\left\{s_{1}, \ldots, s_{n}\right\}$, with $s_{i}= \pm 1$ for all $i$, such that $S \mathcal{L} S$ has all nonpositive off-diagonal entries;

(iii) the smallest eigenvalue of $\mathcal{L}$ is zero, i.e., $\lambda_{1}(\mathcal{L})=0$.

The first condition of Theorem 4.1 corresponds to the original definition of structurally balanced network introduced by Harary and Cartwright [15].

The second condition, illustrated in Fig. 4.3b and Fig. 4.3c, means that applying special transformations, called switching equivalence or signature similarity transformations in the signed graph theory literature [14], it is possible to obtain a graph whose edges have all positive weights. In practice, these transformations consist of changing the signs of all the edges adjacent to a set of nodes, see Fig. 4.3c, which can be identified by the signature matrix $S$ and is given either by $\left\{i \in \mathcal{V}: s_{i}=+1\right\}$ or $\left\{i \in \mathcal{V}: s_{i}=-1\right\}$.

Finally, the third condition can be used to clarify one of the properties of the signed (opposing) Laplacian, see the first line of Table 4.1. Using the Geršgorin's Theorem, it is clear that all the eigenvalues of $\mathcal{L}$ have nonnegative real part. Thanks to Theorem 4.1 it is possible to add that, in particular, 0 is an eigenvalue of $\mathcal{L}$ (with eigenvector $S \mathbb{1}$ ) if and only if the graph is structurally balanced.

When a graph is structurally unbalanced, the question naturally arises as to how far the signed graph is from a structurally balanced state. The standard "measure" of such a distance is the frustration index, hereafter simply denoted frustration, originally introduced by Harary [17, 60] for unweighted signed graphs under the name line index of balance. It indicates the minimum (weighted) sum of the negative edges that have to be removed in order to obtain a graph that is structurally balanced.

Definition 4.3. The frustration $\epsilon(\mathcal{G})$ of a signed graph $\mathcal{G}$ is defined as the minimum (weighted) sum of the positive edges over all signature similarity transformations of $\mathcal{L}$ :

$$
\epsilon(\mathcal{G})=\min _{\substack{S=\operatorname{diag}\left\{s_{1}, \ldots, s_{n}\right\} \\ s_{i}= \pm 1 \forall i}} \frac{1}{2} \sum_{\substack{i, j=1 \\ j \neq i}}^{n}[|\mathcal{L}|+S \mathcal{L} S]_{i j}
$$

Observe that if a signed graph $\mathcal{G}$ is structurally balanced, then $\epsilon(\mathcal{G})=0$ (see e.g., Theorem 4.1(ii)).

Another (maybe more intuitive) interpretation of the notion of frustration, which comes from the statistical physics literature ${ }^{3}$, is that the frustration is the minimum over all possible signature matrices $S$ of an energy functional, defined as

$$
e(S)=\frac{1}{2} \sum_{\substack{i, j=1 \\ j \neq i}}^{n}[|\mathcal{L}|+S \mathcal{L} S]_{i j}
$$

Using this analogy, the frustration quantifies the amount of "disorder" in a signed network.

\footnotetext{
${ }^{3}$ In particular from the Ising spin glass literature, see [18, 61-63], where the frustration is defined as the energy in the ground state of an Ising spin glass.
} 


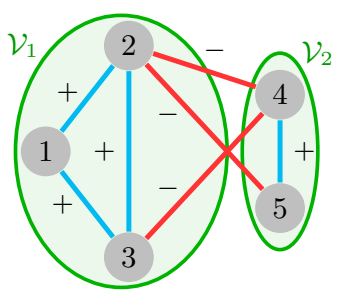

(a)

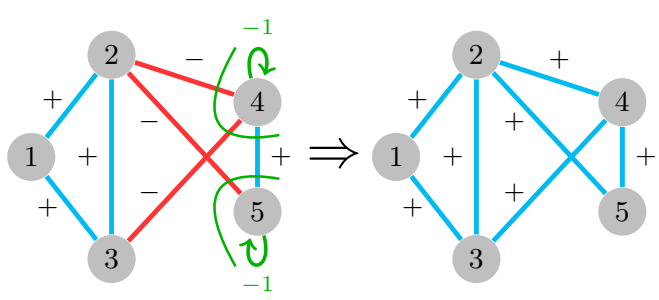

(c)

$$
\mathcal{L}=\left[\begin{array}{rrrrr}
1 & - & - & & \\
- & 1 & - & + & + \\
- & - & 1 & + & \\
+ & + & 1 & - \\
+ & & - & 1
\end{array}\right] \Rightarrow S \mathcal{L} S=\left[\begin{array}{ccccc}
1 & - & - & & \\
- & 1 & - & - & - \\
- & - & 1 & - & \\
- & - & 1 & - \\
- & & - & 1
\end{array}\right]
$$

(b)

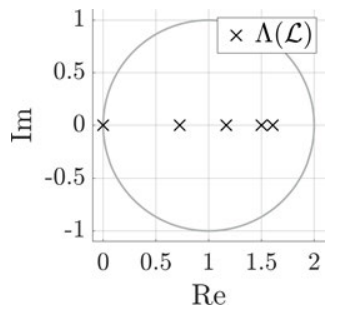

(d)

Figure 4.3: Illustration of the notion of structural balance, see Definition 4.2 and Theorem 4.1. (a): Structurally balanced signed graph. (b): Applying the signature matrix $S=\operatorname{diag}\{1,1,1,-1,-1\}$ to the signed graph in (a) shows that $S \mathcal{L} S$ is a M-matrix. (c): Equivalently, changing the signs of the edges adjacent to nodes 4 and 5 (with $\{4,5\}=\left\{i \in \mathcal{V}: s_{i}=-1\right\}$ ) yields a graph with positive edges. (d) Geršgorin's disks and eigenvalues of $\mathcal{L}$ (computed for the signed graph in (a) with weights $\left.a_{i j} \in\{-1,0,1\}\right)$.

In practice, the computation of the frustration (4.8) is an NP-hard problem. Several algorithms are proposed in the literature, see e.g., [18, 21, 64], and in particular the papers in Part II use the algorithm introduced in [21] to compute numerically the frustration of a signed graph.

Another way to measure the level of imbalance of a signed graph $\mathcal{G}$ is the algebraic conflict, defined simply as the smallest eigenvalue of the normalized signed Laplacian $\mathcal{L}$ of $\mathcal{G}, \lambda_{1}(\mathcal{L})$, which is known to be strictly positive for structurally unbalanced signed graphs (see Theorem 4.1(iii)). In addition, $\lambda_{1}(\mathcal{L})$ is known to approximate well the frustration of a signed network [19, 24, 33], as shown in the following example (inspired by $[28,33])$.

\section{Example 4.1}

In this example a sequence of signed graphs with increasing frustration is considered and, for each graph of the sequence, the values of frustration and of the smallest eigenvalue of the normalized signed Laplacian are computed and compared. The sequence is constructed as follows. Each graph of the sequence $\mathcal{G}=(\mathcal{V}, \mathcal{E}, A)$ has $\operatorname{card}(\mathcal{V})=n=500$ nodes, and the edge weights are drawn from a uniform distribution (where $p=0.8$ is the edge probability). The signature of $\mathcal{G}$ is dependent on a parameter $\beta \in[0,1]$ : in particular, if $(j, i) \in \mathcal{E}$ then $P\left[a_{i j}<0\right]=\beta$. In practice, $\beta$ represents the percentage of edges with negative weight in the signed graph $\mathcal{G}$. 

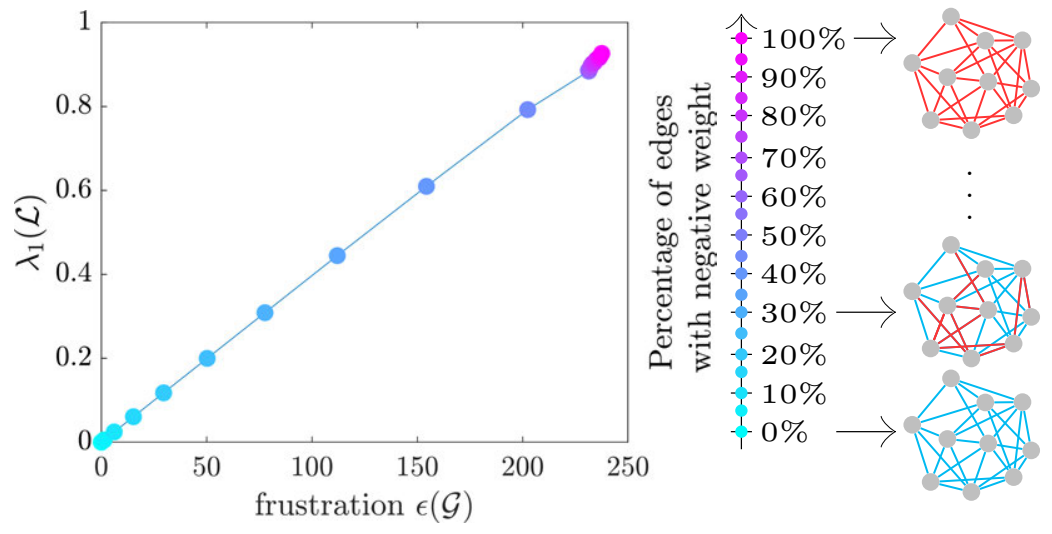

Figure 4.4: Example 4.1. Comparison between smallest eigenvalue of the normalized signed Laplacian $\lambda_{1}(\mathcal{L})$ (i.e., algebraic conflict in the structural balance literature $[24])$ and frustration $\epsilon(\mathcal{G})$, for a sequence of signed graphs with increasing frustration.

The sequence is considered for increasing values of $\beta, \beta \in\{0,0.05, \ldots, 1\}$, and the frustration $\epsilon(\mathcal{G})$ of each signed graph in the sequence, computed numerically using the algorithm proposed in [21], is shown to increase. Figure 4.4 plots the smallest eigenvalue of the normalized signed Laplacian $\mathcal{L}$ vs the frustration, for each signed graph of the sequence. 


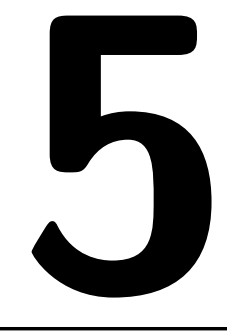

\section{Concluding remarks}

The focus of this thesis is on the study of the collective behavior in a community of agents exchanging opinions, and on understanding how it could be influenced by the presence of antagonistic interactions among the agents.

Several classes of models are considered for this scope. Even though different protocols for consensus have been proposed in the literature, there are cases in which these protocols may prove limiting/restrictive: for instance they fail to represent situations in which the agents may want to rule out particular values of consensus, or to impose that the consensus value respects their own "comfort interval".

For this reason, the first paper of this thesis proposes a new protocol for consensus, denoted interval consensus, which allows the agents to impose constraints on the achievable consensus value. It is a nonlinear interconnected model with a Laplacian-like scheme, where saturations are introduced to describe how the agents transmit their opinions to their neighbors in the network. It is shown that, while during the transient the opinions need not satisfy the constraints imposed by the agents through the saturations, asymptotic convergence to interval consensus is achieved: the opinions converge to a common value belonging to the intersection of the constraints (if nonempty). Formally, existence and asymptotic stability of the equilibrium point of consensus is proven, in case of strongly connected networks. Existence of (at least) an equilibrium point (which, in general, is not a consensus value) is also shown when the intersection is empty, a case which is perhaps less relevant in practical applications.

The second class of models considered in this thesis work, and introduced in paper B, presents a similar design, in that it is characterized by a Laplacian-like structure at the origin, and saturated nonlinearities of sigmoidal type. Differently from the first class of models, the amplitude of the interaction part of the dynamics is modulated by a scalar positive parameter, which plays the role of bifurcation parameter in the analysis. As the parameter is varied, the system exhibits a pitchfork 
bifurcation behavior: for small values of the parameter the origin is the unique equilibrium point of the system and it is globally asymptotically stable. When the parameter increases and crosses a first threshold value, the system undergoes a pitchfork bifurcation and two new alternative (one positive, one negative) equilibria appear, which are locally asymptotically stable, and, in the particular case of identical nonlinearities, are consensus values. The uniqueness of these three equilibria (the origin, and the two alternative consensus equilibria) is proven for all values of the bifurcation parameter less than a second threshold value, after which the system undergoes a second pitchfork bifurcation and new mixed-sign equilibria appear. The equilibrium points that the system admits for high values of the bifurcation parameter may be stable or unstable (as shown by means of numerical simulations), and, while an estimate of their total number is not provided, their norm is shown to be upper bounded by the norm of the (stable) consensus equilibria. Finally, it is shown in paper B that, while the first threshold value for the bifurcation parameter is fixed/constant, the second threshold value depends on the algebraic connectivity of the network, meaning that the "robustness" of the consensus problem (in the sense of size of the interval of values of the bifurcation parameter for which the system admits only three equilibria) is influenced by the network's topology.

The behavior of the system can be interpreted in terms of collective decisionmaking, where the bifurcation parameter represents the "social effort" or "strength of commitment" among the agents. In order to escape from a deadlock situation (where the origin is the only equilibrium point, i.e., no decision is taken) and achieve a decision (i.e., convergence to one of the two alternative nonzero equilibrium points is achieved), the agents need to have the "right" amount of commitment; while this decision represents typically an agreement, disagreement situations are always possible if the agents become "overcommitted" (i.e., the second bifurcation threshold is crossed).

The nonlinear models considered in papers A and B are cooperative, meaning that a decision (in general, of consensus) is reached through collaboration among the agents. This assumption is however restrictive in many real-world scenarios, where rivalry among the agents is inevitable. For this reason, in paper $\mathrm{C}$ the nonlinear model of paper $\mathrm{B}$ is extended to the case of antagonistic networks, by representing the competitive interactions among the agents through a signed network. When the network is structurally balanced, the system is monotone and its behavior is identical to the model of paper B, modulo a change of orthant: instead of consensus, the agents' opinions reach bipartite consensus (i.e., they reach consensus in absolute value). The qualitative behavior of the system does not change also when the network is structurally unbalanced, however the value of the bifurcation parameter for which the first pitchfork bifurcation is crossed is shown to increase with the frustration of the signed network, which represents the amount of social tension present in the community. The interpretation is that the higher is the frustration, the higher is the social commitment required from the agents in order to reach a decision.

This idea is then used in paper D, whose aim is to investigate the complexity of government formation processes in countries with a parliamentary system, 
through an approach based on decision dynamics over signed networks. In particular, by mapping the (post-election) parliament into a signed network, and treating the government formation process as a collective decision-making system over a network (with parliamentary members as agents, and vote of confidence given to the post-election new government as decision), the hypothesis, whose intuition comes from the theoretical analysis of paper $\mathrm{C}$, is that the frustration of the parliamentary network should correlate with the duration of the government negotiation talks: a long period of negotiations is to be expected when the social tension in the parliament is high. This can indeed be seen in the real electoral data in 29 European countries analyzed in paper D. Moreover, it is shown that the concept of frustration of the parliamentary networks can be used also to predict the composition of the successful post-election cabinet coalition.

The work of papers C and D (focused on social/parliamentary networks), where structural balance theory proves significant in order to investigate and interpret the collective behavior of multiagent systems, is one example of the relevance of the study of signed graphs. The focus of paper $\mathrm{E}$ is on the structure of signed digraphs, and in particular of a signed version of Laplacian, denoted "repelling" signed Laplacian, and its pseudoinverse, used for instance in the context of electric networks. Passing from unsigned to signed graphs, important properties of this Laplacian matrix, such as diagonal dominance and marginal stability, are in general no longer guaranteed. Moreover, even in the unsigned case, the set of Laplacian matrices is not closed under pseudoinversion. In paper E, the set of signed Laplacian matrices that are weight balanced and eventually exponentially positive (i.e., matrices whose exponential satisfies the strong Perron-Frobenius property) is shown to be closed not only w.r.t. marginal stability but also to matrix pseudoinversion.

\section{Directions of future research}

This section discusses possible directions in which the results presented in this thesis work could be extended.

Regarding the nonlinear (possibly, antagonistic) model over networks presented in papers $\mathrm{B}$ to $\mathrm{D}$, it is important to observe that the pitchfork bifurcation behavior the multiagent system exhibits is due to symmetry (in the dynamics and in the graph), and an interesting development would be to understand the effect of perturbations or symmetry-breaking phenomena. One direction would be to remove the assumption that the (signed) network representing the community of agents is undirected. In the cooperative or, in general, monotone case (corresponding to a nonnegative or structurally balanced graph) the intuition is that the general behavior of the system would not change: for instance, it would still not admit stable limit cycles. However, this would be no longer true when monotonicity does not hold (i.e., when the signed graph is not structurally balanced): in this case, a Hopf bifurcation might occur and periodic solutions arise. Perhaps more interesting would be to study the effect of adding a constant input to the dynamics, representing for instance external factors, or preference of the agents for a certain alternative. This has been investigated in [4] for the cooperative case, where the 
authors use unfolding theory of a pitchfork bifurcation (see e.g. [48]) to discuss how the preference of the agents for one of the two possible alternatives (represented by two consensus equilibria) affects the choice they make as a group.

Concerning instead the results presented in paper E, while from one side efforts should be spent in trying to characterize better the structure of the set of eventually exponentially positive and weight balanced Laplacian matrices, on the other side it would be interesting to consider the possible applications of the Laplacian pseudoinverse to signed networks: for instance, in the context of electrical networks, by exploring the connection between the signed Laplacian pseudoinverse and the concept of effective resistance, which has been only briefly mentioned in paper E.

The study of theory of eventually (exponentially) positive matrices presented in paper $\mathrm{E}$ (and [57]) could also be beneficial in the context of nonlinear dynamics over signed networks of paper $\mathrm{C}$. Indeed, the way paper $\mathrm{C}$ extends the nonlinear cooperative model presented in paper B to the antagonistic case is to assume that its linearization at the origin corresponds to the version of signed Laplacian denoted "opposing" signed Laplacian. It would be interesting instead to consider the version denoted "repelling" signed Laplacian, and use the work of paper E (and [57]) to investigate the behavior of the corresponding nonlinear model and characterize its equilibria (e.g., if they represent agreement or disagreement); additionally, the results could be compared with the ones obtained for linear systems in [65]. 


\section{Bibliography}

[1] N. E. Leonard, "Multi-agent system dynamics: Bifurcation and behavior of animal groups," Annual Reviews in Control, vol. 38, no. 2, pp. 171-183, 2014.

[2] I. D. Couzin, C. C. Ioannou, G. Demirel, T. Gross, C. J. Torney, A. Hartnett, L. Conradt, S. A. Levin, and N. E. Leonard, "Uninformed individuals promote democratic consensus in animal groups," Science, vol. 334, no. 6062, pp. 1578 1580, 2011.

[3] D. T. Swain, I. D. Couzin, and N. Ehrich Leonard, "Real-Time FeedbackControlled Robotic Fish for Behavioral Experiments With Fish Schools," Proceedings of the IEEE, vol. 100, no. 1, pp. 150-163, jan 2012.

[4] R. Gray, A. Franci, V. Srivastava, and N. E. Leonard, "Multiagent DecisionMaking Dynamics Inspired by Honeybees," IEEE Transactions on Control of Network Systems, vol. 5, no. 2, pp. 793-806, jun 2018.

[5] J. A. Fax and R. M. Murray, "Information Flow and Cooperative Control of Vehicle Formations," IEEE Transactions on Automatic Control, vol. 49, no. 9, pp. 1465-1476, sep 2004.

[6] W. Ren and R. W. Beard, Distributed Consensus in Multi-vehicle Cooperative Control, ser. Communications and Control Engineering. London: Springer London, 2008.

[7] W. Ren and Y. Cao, Distributed Coordination of Multi-agent Networks, ser. Communications and Control Engineering. London: Springer London, 2011.

[8] R. Hegselmann and U. Krause, "Opinion Dynamics and Bounded Confidence," Journal of Artificial Societies and Social Simulation, vol. 5, no. 3, p. 2, 2002.

[9] P. Jia, A. MirTabatabaei, N. E. Friedkin, and F. Bullo, "Opinion Dynamics and the Evolution of Social Power in Influence Networks," SIAM Review, vol. 57 , no. 3, pp. 367-397, 2015.

[10] D. Easley and J. Kleinberg, Networks, Crowds, and Markets: Reasoning about a Highly Connected World. Cambridge: Cambridge University Press, 2010 . 
[11] A. V. Proskurnikov and R. Tempo, "A tutorial on modeling and analysis of dynamic social networks. Part I," Annual Reviews in Control, vol. 43, pp. 65-79, 2017.

[12] A. V. Proskurnikov and R. Tempo, "A tutorial on modeling and analysis of dynamic social networks. Part II," Annual Reviews in Control, vol. 45, pp. 166-190, jan 2018.

[13] W. Ren, R. W. Beard, and E. M. Atkins, "A survey of consensus problems in multi-agent coordination," in 2005 American Control Conference (ACC), vol. 3. IEEE, 2005, pp. 1859-1864.

[14] T. Zaslavsky, "Signed graphs," Discrete Applied Mathematics, vol. 4, no. 1, pp. 47-74, 1982.

[15] D. Cartwright and F. Harary, "Structural balance: a generalization of Heider's theory." Psychological Review, vol. 63, no. 5, pp. 277-293, 1956.

[16] C. Altafini, "Consensus Problems on Networks With Antagonistic Interactions," IEEE Transactions on Automatic Control, vol. 58, no. 4, pp. 935-946, apr 2013.

[17] F. Harary, "On the measurement of structural balance," Behavioral Science, vol. 4, no. 4, pp. 316-323, jan 1959.

[18] G. Facchetti, G. Iacono, and C. Altafini, "Computing global structural balance in large-scale signed social networks," Proceedings of the National Academy of Sciences, vol. 108, no. 52, pp. 20 953-20 958, 2011.

[19] S. Aref and M. C. Wilson, "Measuring partial balance in signed networks," Journal of Complex Networks, vol. 6, no. 4, pp. 566-595, aug 2018.

[20] M. W. Hirsch, S. Smale, and R. L. Devaney, Differential Equations, Dynamical Systems, and an Introduction to Chaos, 2nd ed., A. Press, Ed. Elsevier, 2004.

[21] G. Iacono, F. Ramezani, N. Soranzo, and C. Altafini, "Determining the distance to monotonicity of a biological network: a graph-theoretical approach," IET Systems Biology, vol. 4, no. 3, pp. 223-235, may 2010.

[22] E. D. Sontag, "Monotone and near-monotone biochemical networks," Systems and Synthetic Biology, vol. 1, no. 2, pp. 59-87, 2007.

[23] J. Kunegis, S. Schmidt, A. Lommatzsch, J. Lerner, E. W. De Luca, and S. Albayrak, "Spectral Analysis of Signed Graphs for Clustering, Prediction and Visualization," in 2010 SIAM International Conference on Data Mining. Philadelphia, PA: Society for Industrial and Applied Mathematics, apr 2010, pp. 559-570.

[24] J. Kunegis, "Applications of Structural Balance in Signed Social Networks," arXiv:1402.6865v1, pp. 1-37, 2014. 
[25] A. Fontan and C. Altafini, "A signed network perspective on the government formation process in parliamentary democracies," Scientific Reports, vol. 11, no. 5134, dec 2021.

[26] A. Fontan, G. Shi, X. Hu, and C. Altafini, "Interval Consensus for Multiagent Networks," IEEE Transactions on Automatic Control, vol. 65, no. 5, pp. 18551869 , may 2020.

[27] A. Fontan and C. Altafini, "Multiequilibria Analysis for a Class of Collective Decision-Making Networked Systems," IEEE Transactions on Control of Network Systems, vol. 5, no. 4, pp. 1931-1940, dec 2018.

[28] A. Fontan and C. Altafini, "The role of frustration in collective decision-making dynamical processes on multiagent signed networks," arXiv:2105.11396, pp. 1-18, may 2021.

[29] A. Fontan and C. Altafini, "On the properties of Laplacian pseudoinverses," in 60th IEEE Conference on Decision and Control. Austin, TX, USA: IEEE, 2021.

[30] A. Fontan, G. Shi, X. Hu, and C. Altafini, "Interval consensus: a novel class of constrained consensus problems for multiagent networks," in 56th IEEE Conference on Decision and Control. Melbourne, Australia: IEEE, dec 2017, pp. $4155-4160$.

[31] A. Fontan and C. Altafini, "Investigating mixed-sign equilibria for nonlinear collective decision-making systems," in 56th IEEE Conference on Decision and Control. Melbourne, Australia: IEEE, dec 2017, pp. 781-786.

[32] A. Fontan and C. Altafini, "Modeling wireless power transfer in a network of smart devices: A compartmental system approach," in 2018 17th European Control Conference (ECC). Limassol, Cyprus: European Control Association (EUCA), 2018, pp. 1468-1473.

[33] A. Fontan and C. Altafini, "Achieving a decision in antagonistic multi agent networks: frustration determines commitment strength," in 57th IEEE Conference on Decision and Control. Miami Beach, FL, USA: IEEE, dec 2018, pp. 109-114.

[34] R. A. Horn and C. R. Johnson, Matrix analysis, 2nd ed. Cambridge University Press, 2013.

[35] E. Kaszkurewicz and A. Bhaya, Matrix Diagonal Stability in Systems and Computation. Boston: Birkhäuser, 2000.

[36] S. Camiz and S. Stefani, Matrices and Graphs. World Scientific, 1996.

[37] C. Altafini, "Stability analysis of diagonally equipotent matrices," Automatica, vol. 49, no. 9, pp. 2780-2785, sep 2013. 
[38] D. Noutsos, "On Perron-Frobenius property of matrices having some negative entries," Linear Algebra and its Applications, vol. 412, no. 2-3, pp. 132-153, jan 2006.

[39] A. Berman and R. J. Plemmons, Nonnegative Matrices in the Mathematical Sciences, ser. Classic in applied mathematics. Society for Industrial and Applied Mathematics, 1994.

[40] H. K. Khalil, Nonlinear Systems: Third Edition. Pearson, 2002.

[41] M. W. Hirsch, "Systems of Differential Equations that are Competitive or Cooperative II: Convergence Almost Everywhere," SIAM Journal on Mathematical Analysis, vol. 16, no. 3, pp. 423-439, may 1985.

[42] H. L. Smith, "Systems of Ordinary Differential Equations Which Generate an Order Preserving Flow. A Survey of Results," SIAM Review, vol. 30, no. 1, pp. 87-113, mar 1988.

[43] M. W. Hirsch and H. L. Smith, "Monotone Dynamical Systems," in Handbook of Differential Equations: Ordinary Differential Equations. North-Holland, 2006, vol. 2, ch. 4, pp. 239-357.

[44] N. Rouche, P. Habets, and M. Laloy, Stability Theory by Liapunov's Direct Method. Springer New York, 1977.

[45] L. Perko, Differential Equations and Dynamical Systems, ser. Texts in Applied Mathematics. New York, NY: Springer New York, 2001, vol. 7.

[46] Y. A. Kuznetsov, Elements of Applied Bifurcation Theory, Second Edition. Springer, 1998.

[47] F. Blanchini and S. Miani, Set-Theoretic Methods in Control, ser. Systems \& Control: Foundations \& Applications. Boston, MA: Birkhäuser Boston, 2008 .

[48] M. Golubitsky and D. G. Schaeffer, Singularities and Groups in Bifurcation Theory. Volume I, ser. Applied Mathematical Sciences. Springer New York, 1985, vol. 51 .

[49] M. Golubitsky and I. Stewart, The Symmetry Perspective, ser. Progress in Mathematics (Volume 200), H. Bass, J. Oesterle, and A. Weinstein, Eds. Basel: Birkhäuser Basel, 2002.

[50] C. Godsil and G. Royle, Algebraic Graph Theory, ser. Graduate Texts in Mathematics. New York, NY: Springer New York, 2001, vol. 207.

[51] F. R. K. Chung, Spectral Graph Theory, ser. CBMS Number 92. American Mathematical Society, 1997.

[52] F. Bullo, Lectures on Nonlinear Network Systems (ed. 1.4). Kindle Direct Publishing, 2020. http://motion.me.ucsb.edu/book-lns 
[53] T. Zaslavsky, "Matrices in the Theory of Signed Simple Graphs," arXiv:1303.3083v1, pp. 1-20, mar 2013.

[54] G. Shi, C. Altafini, and J. S. Baras, "Dynamics over Signed Networks," SIAM Review, vol. 61, no. 2, pp. 229-257, jan 2019.

[55] R. Olfati-Saber and R. Murray, "Consensus Problems in Networks of Agents With Switching Topology and Time-Delays," IEEE Transactions on Automatic Control, vol. 49, no. 9, pp. 1520-1533, sep 2004.

[56] R. Agaev and P. Chebotarev, "On the spectra of nonsymmetric Laplacian matrices," Linear Algebra and Its Applications, vol. 399, no. 1-3, pp. 157$168,2005$.

[57] C. Altafini, "Investigating stability of Laplacians on signed digraphs via eventual positivity," in 58th IEEE Conference on Decision and Control. Nice, France: IEEE, dec 2019, pp. 5044-5049.

[58] F. Heider, "Attitudes and Cognitive Organization," The Journal of Psychology, vol. 21, no. 1, pp. 107-112, jan 1946.

[59] F. Harary, "On the notion of balance of a signed graph." The Michigan Mathematical Journal, vol. 2, no. 2, pp. 143-146, 1953.

[60] F. Harary and J. A. Kabell, "A simple algorithm to detect balance in signed graphs," Mathematical Social Sciences, vol. 1, no. 1, pp. 131-136, sep 1980.

[61] F. Barahona, "On the computational complexity of ising spin glass models," Journal of Physics A: Mathematical and General, vol. 15, no. 10, pp. 32413253, 1982.

[62] K. Binder and A. P. Young, "Spin glasses: Experimental facts, theoretical concepts, and open questions," Reviews of Modern Physics, vol. 58, no. 4, pp. 801-976, oct 1986 .

[63] M. Mézard and G. Parisi, "The Bethe lattice spin glass revisited," The European Physical Journal B, vol. 20, no. 2, pp. 217-233, mar 2001.

[64] S. Aref, A. J. Mason, and M. C. Wilson, "An exact method for computing the frustration index in signed networks using binary programming," arXiv:1611.09030v2, nov 2016.

[65] C. Altafini and G. Lini, "Predictable dynamics of opinion forming for networks with antagonistic interactions," IEEE Transactions on Automatic Control, vol. 60, no. 2, pp. 342-357, feb 2015 . 

Part II

Publications 


\section{Papers}

The papers associated with this thesis have been removed for copyright reasons. For more details about these see:

http://urn.kb.se/resolve?urn=urn:nbn:se:liu:diva-178301 


\section{PhD Dissertations \\ Division of Automatic Control \\ Linköping University}

M. Millnert: Identification and control of systems subject to abrupt changes. Thesis No. 82, 1982. ISBN 91-7372-542-0.

A. J. M. van Overbeek: On-line structure selection for the identification of multivariable systems. Thesis No. 86, 1982. ISBN 91-7372-586-2.

B. Bengtsson: On some control problems for queues. Thesis No. 87, 1982. ISBN 917372-593-5.

S. Ljung: Fast algorithms for integral equations and least squares identification problems. Thesis No. 93, 1983. ISBN 91-7372-641-9.

H. Jonson: A Newton method for solving non-linear optimal control problems with general constraints. Thesis No. 104, 1983. ISBN 91-7372-718-0.

E. Trulsson: Adaptive control based on explicit criterion minimization. Thesis No. 106, 1983. ISBN 91-7372-728-8.

K. Nordström: Uncertainty, robustness and sensitivity reduction in the design of single input control systems. Thesis No. 162, 1987. ISBN 91-7870-170-8.

B. Wahlberg: On the identification and approximation of linear systems. Thesis No. 163, 1987. ISBN 91-7870-175-9.

S. Gunnarsson: Frequency domain aspects of modeling and control in adaptive systems. Thesis No. 194, 1988. ISBN 91-7870-380-8.

A. Isaksson: On system identification in one and two dimensions with signal processing applications. Thesis No. 196, 1988. ISBN 91-7870-383-2.

M. Viberg: Subspace fitting concepts in sensor array processing. Thesis No. 217, 1989. ISBN 91-7870-529-0.

K. Forsman: Constructive commutative algebra in nonlinear control theory. Thesis No. 261, 1991. ISBN 91-7870-827-3.

F. Gustafsson: Estimation of discrete parameters in linear systems. Thesis No. 271, 1992. ISBN 91-7870-876-1.

P. Nagy: Tools for knowledge-based signal processing with applications to system identification. Thesis No. 280, 1992. ISBN 91-7870-962-8.

T. Svensson: Mathematical tools and software for analysis and design of nonlinear control systems. Thesis No. 285, 1992. ISBN 91-7870-989-X.

S. Andersson: On dimension reduction in sensor array signal processing. Thesis No. 290, 1992. ISBN 91-7871-015-4.

H. Hjalmarsson: Aspects on incomplete modeling in system identification. Thesis No. 298, 1993. ISBN 91-7871-070-7.

I. Klein: Automatic synthesis of sequential control schemes. Thesis No. 305, 1993. ISBN 91-7871-090-1.

J.-E. Strömberg: A mode switching modelling philosophy. Thesis No. 353, 1994. ISBN 91-7871-430-3.

K. Wang Chen: Transformation and symbolic calculations in filtering and control. Thesis No. 361, 1994. ISBN 91-7871-467-2.

T. McKelvey: Identification of state-space models from time and frequency data. Thesis No. 380, 1995. ISBN 91-7871-531-8.

J. Sjöberg: Non-linear system identification with neural networks. Thesis No. 381, 1995. ISBN 91-7871-534-2.

R. Germundsson: Symbolic systems - theory, computation and applications. Thesis No. 389, 1995. ISBN 91-7871-578-4. 
P. Pucar: Modeling and segmentation using multiple models. Thesis No. 405, 1995. ISBN 91-7871-627-6.

H. Fortell: Algebraic approaches to normal forms and zero dynamics. Thesis No. 407, 1995. ISBN 91-7871-629-2.

A. Helmersson: Methods for robust gain scheduling. Thesis No. 406, 1995. ISBN 917871-628-4.

P. Lindskog: Methods, algorithms and tools for system identification based on prior knowledge. Thesis No. 436, 1996. ISBN 91-7871-424-8.

J. Gunnarsson: Symbolic methods and tools for discrete event dynamic systems. Thesis No. 477, 1997. ISBN 91-7871-917-8.

M. Jirstrand: Constructive methods for inequality constraints in control. Thesis No. 527, 1998. ISBN 91-7219-187-2.

U. Forssell: Closed-loop identification: Methods, theory, and applications. Thesis No. 566, 1999. ISBN 91-7219-432-4.

A. Stenman: Model on demand: Algorithms, analysis and applications. Thesis No. 571, 1999. ISBN 91-7219-450-2.

N. Bergman: Recursive Bayesian estimation: Navigation and tracking applications. Thesis No. 579, 1999. ISBN 91-7219-473-1.

K. Edström: Switched bond graphs: Simulation and analysis. Thesis No. 586, 1999. ISBN 91-7219-493-6.

M. Larsson: Behavioral and structural model based approaches to discrete diagnosis. Thesis No. 608, 1999. ISBN 91-7219-615-5.

F. Gunnarsson: Power control in cellular radio systems: Analysis, design and estimation. Thesis No. 623, 2000. ISBN 91-7219-689-0.

V. Einarsson: Model checking methods for mode switching systems. Thesis No. 652, 2000. ISBN 91-7219-836-2.

M. Norrlöf: Iterative learning control: Analysis, design, and experiments. Thesis No. 653, 2000. ISBN 91-7219-837-0.

F. Tjärnström: Variance expressions and model reduction in system identification. Thesis No. 730, 2002. ISBN 91-7373-253-2.

J. Löfberg: Minimax approaches to robust model predictive control. Thesis No. 812, 2003. ISBN 91-7373-622-8.

J. Roll: Local and piecewise affine approaches to system identification. Thesis No. 802, 2003. ISBN 91-7373-608-2.

J. Elbornsson: Analysis, estimation and compensation of mismatch effects in A/D converters. Thesis No. 811, 2003. ISBN 91-7373-621-X.

O. Härkegård: Backstepping and control allocation with applications to flight control. Thesis No. 820, 2003. ISBN 91-7373-647-3.

R. Wallin: Optimization algorithms for system analysis and identification. Thesis No. 919, 2004. ISBN 91-85297-19-4.

D. Lindgren: Projection methods for classification and identification. Thesis No. 915, 2005. ISBN 91-85297-06-2.

R. Karlsson: Particle Filtering for Positioning and Tracking Applications. Thesis No. 924, 2005. ISBN 91-85297-34-8.

J. Jansson: Collision Avoidance Theory with Applications to Automotive Collision Mitigation. Thesis No. 950, 2005. ISBN 91-85299-45-6.

E. Geijer Lundin: Uplink Load in CDMA Cellular Radio Systems. Thesis No. 977, 2005. ISBN 91-85457-49-3.

M. Enqvist: Linear Models of Nonlinear Systems. Thesis No. 985, 2005. ISBN 9185457-64-7. 
T. B. Schön: Estimation of Nonlinear Dynamic Systems - Theory and Applications. Thesis No. 998, 2006. ISBN 91-85497-03-7.

I. Lind: Regressor and Structure Selection - Uses of ANOVA in System Identification. Thesis No. 1012, 2006. ISBN 91-85523-98-4.

J. Gillberg: Frequency Domain Identification of Continuous-Time Systems Reconstruction and Robustness. Thesis No. 1031, 2006. ISBN 91-85523-34-8.

M. Gerdin: Identification and Estimation for Models Described by DifferentialAlgebraic Equations. Thesis No. 1046, 2006. ISBN 91-85643-87-4.

C. Grönwall: Ground Object Recognition using Laser Radar Data - Geometric Fitting, Performance Analysis, and Applications. Thesis No. 1055, 2006. ISBN 91-85643-53-X.

A. Eidehall: Tracking and threat assessment for automotive collision avoidance. Thesis No. 1066, 2007. ISBN 91-85643-10-6.

F. Eng: Non-Uniform Sampling in Statistical Signal Processing. Thesis No. 1082, 2007. ISBN 978-91-85715-49-7.

E. Wernholt: Multivariable Frequency-Domain Identification of Industrial Robots. Thesis No. 1138, 2007. ISBN 978-91-85895-72-4.

D. Axehill: Integer Quadratic Programming for Control and Communication. Thesis No. 1158, 2008. ISBN 978-91-85523-03-0.

G. Hendeby: Performance and Implementation Aspects of Nonlinear Filtering. Thesis No. 1161, 2008. ISBN 978-91-7393-979-9.

J. Sjöberg: Optimal Control and Model Reduction of Nonlinear DAE Models. Thesis No. 1166, 2008. ISBN 978-91-7393-964-5.

D. Törnqvist: Estimation and Detection with Applications to Navigation. Thesis No. 1216, 2008. ISBN 978-91-7393-785-6.

P-J. Nordlund: Efficient Estimation and Detection Methods for Airborne Applications. Thesis No. 1231, 2008. ISBN 978-91-7393-720-7.

H. Tidefelt: Differential-algebraic equations and matrix-valued singular perturbation. Thesis No. 1292, 2009. ISBN 978-91-7393-479-4.

H. Ohlsson: Regularization for Sparseness and Smoothness - Applications in System Identification and Signal Processing. Thesis No. 1351, 2010. ISBN 978-91-7393-287-5.

S. Moberg: Modeling and Control of Flexible Manipulators. Thesis No. 1349, 2010. ISBN 978-91-7393-289-9.

J. Wallén: Estimation-based iterative learning control. Thesis No. 1358, 2011. ISBN 978-91-7393-255-4.

J. D. Hol: Sensor Fusion and Calibration of Inertial Sensors, Vision, Ultra-Wideband and GPS. Thesis No. 1368, 2011. ISBN 978-91-7393-197-7.

D. Ankelhed: On the Design of Low Order H-infinity Controllers. Thesis No. 1371, 2011. ISBN 978-91-7393-157-1.

C. Lundquist: Sensor Fusion for Automotive Applications. Thesis No. 1409, 2011. ISBN 978-91-7393-023-9.

P. Skoglar: Tracking and Planning for Surveillance Applications. Thesis No. 1432, 2012. ISBN 978-91-7519-941-2.

K. Granström: Extended target tracking using PHD filters. Thesis No. 1476, 2012. ISBN 978-91-7519-796-8.

C. Lyzell: Structural Reformulations in System Identification. Thesis No. 1475, 2012. ISBN 978-91-7519-800-2.

J. Callmer: Autonomous Localization in Unknown Environments. Thesis No. 1520, 2013. ISBN 978-91-7519-620-6.

D. Petersson: A Nonlinear Optimization Approach to H2-Optimal Modeling and Control. Thesis No. 1528, 2013. ISBN 978-91-7519-567-4. 
Z. Sjanic: Navigation and Mapping for Aerial Vehicles Based on Inertial and Imaging Sensors. Thesis No. 1533, 2013. ISBN 978-91-7519-553-7.

F. Lindsten: Particle Filters and Markov Chains for Learning of Dynamical Systems. Thesis No. 1530, 2013. ISBN 978-91-7519-559-9.

P. Axelsson: Sensor Fusion and Control Applied to Industrial Manipulators. Thesis No. 1585, 2014. ISBN 978-91-7519-368-7.

A. Carvalho Bittencourt: Modeling and Diagnosis of Friction and Wear in Industrial Robots. Thesis No. 1617, 2014. ISBN 978-91-7519-251-2.

M. Skoglund: Inertial Navigation and Mapping for Autonomous Vehicles. Thesis No. 1623, 2014. ISBN 978-91-7519-233-8.

S. Khoshfetrat Pakazad: Divide and Conquer: Distributed Optimization and Robustness Analysis. Thesis No. 1676, 2015. ISBN 978-91-7519-050-1.

T. Ardeshiri: Analytical Approximations for Bayesian Inference. Thesis No. 1710, 2015. ISBN 978-91-7685-930-8.

N. Wahlström: Modeling of Magnetic Fields and Extended Objects for Localization Applications. Thesis No. 1723, 2015. ISBN 978-91-7685-903-2.

J. Dahlin: Accelerating Monte Carlo methods for Bayesian inference in dynamical models. Thesis No. 1754, 2016. ISBN 978-91-7685-797-7.

M. Kok: Probabilistic modeling for sensor fusion with inertial measurements. Thesis No. 1814, 2016. ISBN 978-91-7685-621-5.

J. Linder: Indirect System Identification for Unknown Input Problems: With Applications to Ships. Thesis No. 1829, 2017. ISBN 978-91-7685-588-1.

M. Roth: Advanced Kalman Filtering Approaches to Bayesian State Estimation. Thesis No. 1832, 2017. ISBN 978-91-7685-578-2.

I. Nielsen: Structure-Exploiting Numerical Algorithms for Optimal Control. Thesis No. 1848, 2017. ISBN 978-91-7685-528-7.

D. Simon: Fighter Aircraft Maneuver Limiting Using MPC: Theory and Application. Thesis No. 1881, 2017. ISBN 978-91-7685-450-1.

C. Veibäck: Tracking the Wanders of Nature. Thesis No. 1958, 2018. ISBN 978-917685-200-2.

C. Andersson Naesseth: Machine learning using approximate inference: Variational and sequential Monte Carlo methods. Thesis No. 1969, 2018. ISBN 978-91-7685-161-6.

Y. Jung: Inverse system identification with applications in predistortion. Thesis No. 1966, 2018. ISBN 978-91-7685-171-5.

Y. Zhao: Gaussian Processes for Positioning Using Radio Signal Strength Measurements. Thesis No. 1968, 2019. ISBN 978-91-7685-162-3.

R. Larsson: Flight Test System Identification. Thesis No. 1990, 2019. ISBN 978-917685-070-1.

P. Kasebzadeh: Learning Human Gait. Thesis No. 2012, 2019. ISBN 978-91-7519-0143.

K. Radnosrati: Time of flight estimation for radio network positioning. Thesis No. 2054, 2020. ISBN 978-91-7929-884-5.

O. Ljungqvist: Motion planning and feedback control techniques with applications to long tractor-trailer vehicles. Thesis No. 2070, 2020. ISBN 978-91-7929-858-6.

G. Lindmark: Controllability of Complex Networks at Minimum Cost. Thesis No. 2074, 2020. ISBN 978-91-7929-847-0.

K. Bergman: Exploiting Direct Optimal Control for Motion Planning in Unstructured Environments. Thesis No. 2133, 2021. ISBN 978-91-7929-677-3.

P. Boström-Rost: Sensor Management for Target Tracking Applications. Thesis No. 2137, 2021. ISBN 978-91-7929-672-8. 


\section{FACULTY OF SCIENCE AND ENGINEERING}

Linköping Studies in Science and Technology, Dissertation No. 2166, 2021

Department of Electrical Engineering

Linköping University

SE-581 83 Linköping, Sweden

www.liu.se 